\title{
A mode shape assembly algorithm by using two stage Bayesian Fast Fourier Transform Approach
}

\author{
Çağlayan Hızal*, Gürsoy Turan, Engin Aktaş, Hasan Ceylan \\ Department of Civil Engineering, Engineering Faculty of Izmir Institute of Technology, 35430 Urla, Izmir, Turkey
}

\section{A R T I C L E I N F O}

\section{Article history:}

Received 31 January 2019

Received in revised form 17 June 2019

Accepted 26 August 2019

Available online 6 September 2019

\section{Keywords:}

BFFTA

Mode shape assembly

Modal identification

Operational modal analysis

Multiple setups

\begin{abstract}
A B S T R A C T
Operational modal analysis may require identifying global modal shapes by using multiple setup measurements. For this purpose, various algorithms have been developed which make use of the Bayesian approach to estimate the global mode shapes. The main motivation of the available Bayesian approaches is based on the estimation of the optimal global mode shape vector directly from Fast Fourier Transform data or assembling the local mode shapes that are identified in the individual setups by using Gaussian approximation. In this study, the two-stage Bayesian Fast Fourier Transform Approach which is originally applied to single setups is implemented to multiple setup problems for well separated modes. Analytically it is shown that the resulting formulation is the same for the mode shape assembly by using the Gaussian approximation. In addition, the weights of individual setups in the global mode shape vector is analytically calculated which depend on the Hessian matrix for local mode shapes. To validate the proposed methodology, a numerical example that considers setup-to-setup variability of modal signal-noise ratios is presented. For comparison purposes a ten-story shear frame model is experimentally investigated, and the measurements of a benchmark bridge structure are considered in the verification of the current procedure.
\end{abstract}

(c) 2019 Elsevier Ltd. All rights reserved.

\section{Introduction}

Modal parameter identification has a key importance in structural health monitoring and damage detection. Modal parameters that are required for structural assessment include natural frequencies, damping ratios and modal shape vectors. In addition to these, spectral density level of excitation and possible measurement errors might be important in the identification process. In this context, Bayesian Operational Modal Analysis (BAYOMA) in frequency domain proposes simple and fast algorithms based on the statistical properties of measured data. Katafygiotis and Yuen [1] first proposed the Bayesian Spectral Density Approach (BSDA) based on the statistical properties of spectral density matrix. A Bayesian Fast Fourier Transform Approach (BFFTA) was proposed by Yuen and Katafygiotis [2] to estimate the most probable modal parameters by using the Fast Fourier Transform (FFT) data. Au [3] proposed a fast algorithm for BFFTA for systems with well separated modes and reformulated the general methodology presented by Yuen and Katafygiotis [2]. In past decade, numerous studies concerning the general computational process and application of Bayesian Spectral Density and Fast Fourier Transform Approach have been presented by Au et al. [4-9]. In addition, Yan and Katafygiotis [10] presented a two stage BSDA that separates the modal parameters to be identified into two components: $(i)$ spectrum variables including frequency, damping

\footnotetext{
* Corresponding author.

E-mail address: caglayanhizal@iyte.edu.tr (Ç. Hızal).
} 
ratio, and spectral density of modal excitation and prediction error, (ii) spatial parameters including the modal shape components. Although BSDA and BFFTA are motivated by different statistical properties of measured data, they result in similar estimators. Various studies have been presented in the literature conducted with the application of BFFTA and BSDA [11-14].

In large scale structures, monitoring of the change in modal parameters has a direct effect to make decisions after extreme events (such as earthquakes, tornadoes, etc.). For this reason, a full scaled monitoring of change in modal parameters becomes necessary. In the literature, it is known that the modal parameter estimation process in large scaled structures requires more computational effort due to the insufficient number of measurement instruments (such as accelerometer, data acquisition devices). This case makes it necessary to use multiple measurement setups in the identification process. Even though the natural frequencies and damping ratios can be obtained via the individual setups, obtaining the global mode shape that covers the all measurement setups arises as an issue. Essentially, there are two different methods in the literature to obtain the global mode shape vector; (i) Pre-identification, and (ii) Post identification. Pre-identification methods are based on various scaling processes to obtain a full set of synchronous measured data. In case of constant reference sensor (or sensors) and large signal-to-noise ratio, pre-identification methods are capable of obtaining the global mode shapes with an acceptable accuracy. However, the convergence of those methods reduces in case of roving sensors and/or relatively low signal levels due to the accumulated errors in scaling procedure $[15,16]$.

Post identification methods are based on the assembly of local mode shapes that are identified in individual setups or direct estimation of global mode shapes incorporated by multiple setups. Here, the local least square approach comes forward as the most conventional one, but the results are highly sensitive to sensor configuration and estimation quality of local mode shapes. More efficient methods have been presented in the current decade for global mode shape assembly or estimation. Au [17] presented the Global Least Squares Approach for the assembly of local mode shapes with an iterative algorithm. This iterative algorithm is based on the minimization of the equally weighted discrepancies between the local part of optimal global mode shape and identified local mode shape. In addition, some probabilistic approaches for global mode shape assembly have also been developed based on the Bayesian inference. Those methods are capable of identifying not only the global mode shapes but also their posterior uncertainties. There are two different approaches in the literature for Bayesian mode shape assembly. First, Au and Zhang [18] presented a Bayesian approach for direct estimation of global mode shape vector and local spectrum variables incorporating the multiple measurement setups. Au [19] modified the original algorithm that was presented by Au and Zhang [18] and proposed a simplified iterative algorithm. In addition, a different technique was presented by Yan and Katafygiotis [20] that is motivated by the Global Least Squares Approach. Their approach is based on the assembling local mode shapes that are identified at individual setups by using Bayesian statistical framework.

The available Bayesian global mode shape identification methods [18-20] are motivated from different approaches (BFFTA and BSDA) and follow different ways to estimate the most probable global mode shape. The basic motivation of this study is to develop an alternative mode shape assembly algorithm which results in same eigenvalue equations (for global mode shapes) that can be obtained by the aforementioned procedures. For this purpose, first, the two-stage BFFTA by Au [19] for single setup problems with well separated modes is adopted to multiple setups and a modified likelihood function for the global mode shape is derived. Second, the solution is verified by the Gaussian approximation for the most probable local mode shape vector. Finally, the weights of individual setups in the most probable global mode shape vector is obtained by using the expansion of Hessian matrix for local mode shapes. The final form of the proposed procedure does not need to identify local mode shapes separately. Instead, it utilizes the statistical information of Hessian matrix of the local mode shapes which can be derived by using only the spectrum variables and FFT data. According to the obtained results, it is seen that the proposed algorithm results in higher convergence speed when compared to the previous BFFTA algorithm.

\section{Two-stage BFFTA for individual setups}

Based on the statistical properties of FFT of the measured acceleration data, the conditional probability density function for the set of modal parameters in an individual setup, $i$, can be written as follows by using the Bayes' theorem [3].

$$
p\left(\theta_{i} \mid Z_{k i}\right)=p\left(Z_{k i} \mid \theta_{i}\right) p\left(\theta_{i}\right) / p\left(Z_{k i}\right)
$$

where $\theta_{i}=\left[f_{i}, \xi_{i}, \bar{S}_{i}, S_{\mathrm{ei}}, \varphi_{i}\right]$, is the set of modal parameters to be identified and comprises the natural frequency, damping ratio, spectral density of modal excitation that is scaled with respect to the unit norm for local mode shape, spectral density of prediction error, and local mode shape vector (with unit norm) at $i^{\text {th }}$ setup, respectively. $\operatorname{Here}, Z_{k i}=\left[\operatorname{Re}\left(F_{k i}\right) ; \operatorname{Im}\left(F_{k i}\right)\right] \epsilon \mathfrak{R}$ denotes the augmented FFT vector of the measured response at the $i^{\text {th }}$ setup. In addition, $p\left(\theta_{i} \mid Z_{k i}\right)=$ the conditional posterior probability density function (pdf) of $\theta_{i}$ for a given $Z_{k i}, p\left(Z_{k i} \mid \theta_{i}\right)=$ the likelihood function which is obtained by a proper probability distribution of $Z_{k i}$ for a given $\theta_{i}, p\left(\theta_{i}\right)$ and $p\left(Z_{k i}\right)$ are prior pdf of $\theta_{i}$ and $Z_{k i}$, respectively. Since, $p\left(Z_{k i}\right)$ does not depend on $\theta_{i}$, it corresponds to a scalar value and can be assumed as a normalizing constant. In the applications of BAYOMA, $p\left(\theta_{i} \mid Z_{k i}\right)$ is generally assumed to be proportional to $p\left(Z_{k i} \mid \theta_{i}\right)$ in case of a non-informative prior distribution, $p\left(\theta_{i}\right)[1,2,3,19]$. In addition, $p\left(\theta_{i} \mid Z_{k i}\right)$ follows a zero mean Gaussian distribution with a large amount of measured data [19].

$$
p\left(\theta_{i} \mid Z_{k i}\right) \propto(2 \pi)^{-N_{i}}\left|C_{\theta_{i}}\right|^{-1} \exp \left(-\frac{1}{2} Z_{k i}^{T} C_{\theta_{i}}^{-1} Z_{k i}\right)
$$

Here, $N_{i}=$ number of measured DOF, $C_{\theta_{i}}=$ expected value of the covariance matrix, $E\left[Z_{k i} Z_{k i}^{T}\right][19]$. 


$$
\begin{aligned}
& E\left[Z_{k i} Z_{k i}^{T}\right]=\frac{1}{2}\left[\begin{array}{cc}
E\left[\operatorname{Re}\left(F_{k i} F_{k i}^{*}\right)\right] & 0 \\
0 & E\left[\operatorname{Re}\left(F_{k i} F_{k i}^{*}\right)\right]
\end{array}\right] \\
& E\left[\operatorname{Re}\left(F_{k i} F_{k i}^{*}\right)\right]=\bar{S}_{i} D_{k i} \varphi_{i} \varphi_{i}^{T}+S_{e i} I_{N i} ; \quad \bar{S}_{i}=r_{i}^{2} S_{i} ; r_{i}= \pm\left\|\psi_{\mathrm{i}}\right\| ; \psi_{i}=r_{i} \varphi_{i}
\end{aligned}
$$

where $[.]^{*}=$ conjugate transpose, $\|\|=$. Euclidian norm, $F_{k i}=$ FFT of measured response at $i^{\text {th }}$ setup, $S_{i}=$ spectral density of modal excitation that is scaled with respect to the unit norm of global mode shape vector, $\psi_{i}=$ local mode shape with norm of $r_{i}$, and $I_{N i}=N_{i} \times N_{i}$ size identity matrix. In addition, $D_{k i}$ is written as below.

$$
D_{k i}=\left[\left(1-\beta_{k i}^{2}\right)^{2}+\left(2 \xi_{i} \beta_{k i}\right)^{2}\right]^{-1} ; \beta_{k i}=f_{i} / f_{k}
$$

Here, $f_{k}=$ excitation frequency. The set of modal parameters can be considered as the combination of local spectrum parameters, $\theta_{s i}=\left[f_{i}, \xi_{i}, \bar{S}_{\mathrm{i}}, S_{\mathrm{ei}}\right]$, and local mode shape vector, $\varphi_{i}$. Thus, the negative logarithm-likelihood function for $p\left(\theta_{i} \mid Z_{k i}\right)$ can be considered in two parts for well separated modes: (i) the first part only is sensitive to $\theta_{s i}$, and (ii) the second part is sensitive to $\theta_{i}[19]$.

$$
\begin{gathered}
L_{i}\left(\theta_{i}\right)=\underbrace{N_{i} N_{f i} \ln \pi+\left(N_{f i}-1\right) \ln S_{e i}+\sum_{k} \ln \left(\bar{S}_{i} D_{k i}+S_{e i}\right)+S_{e i}^{-1} \kappa_{i}}_{\text {First Part }}-\underbrace{\varphi_{i}^{T} \Delta_{i} \varphi_{i}}_{\text {Second Part }} \\
\kappa_{i}=\sum_{k} F_{k i}^{*} F_{k i} ; \quad \Delta_{i}=\sum_{k} \frac{\bar{S}_{i} D_{k i} S_{e i}^{-1}}{\left(\bar{S}_{i} D_{k i}+S_{e i}\right)} \operatorname{Re}\left(F_{k i} F_{k i}^{*}\right)
\end{gathered}
$$

where $N_{f i}=$ number of data within the selected dominant frequency band at $i^{\text {th }}$ setup, respectively. Here, the Most Probable Value (MPV) for each parameter can be determined by the direct minimization of Eq. (5). In this minimization process, the modal parameters including spectral parameters and mode shapes can be decoupled for computational simplicity $[10,19]$. For globally identifiable problems, it is known that Eq. (5) has a unique minimum at the MPV of $\theta_{i}$. For this reason, when the MPV of the local mode shape is considered, the second part of Eq. (5) can be represented as the function of $\theta_{s i}$.

$$
\hat{\varphi}_{i}^{T} \Delta_{i} \hat{\varphi}_{i}=\ell_{i}\left(\theta_{s i}\right)
$$

Here, $\widehat{\varphi}_{i}=$ MPV of local mode shape with unit norm (for $i^{\text {th }}$ setup). Thus, the negative logarithm-likelihood function will only depend on the spectral parameters when $\ell_{i}\left(\theta_{s i}\right)$ is substituted into Eq. (5).

$$
\begin{aligned}
& L_{i}\left(\theta_{i}\right)=L_{s i}\left(\theta_{s i}\right)-\ell_{i}\left(\theta_{s i}\right) \\
& L_{s i}\left(\theta_{s i}\right)=N_{i} N_{f i} \ln \pi+\left(N_{f i}-1\right) \ln S_{e}+\sum_{k} \ln \left(\bar{S}_{i} D_{k i}+S_{e}\right)+S_{e}^{-1} \kappa_{i}
\end{aligned}
$$

The spectral parameters can be obtained by minimizing Eq. (7). It follows that the first part will be constant when the MPV of $\theta_{s i}$ is substituted into Eq. (5). An inclusion of a unit norm constraint for $\varphi_{i}$ to the likelihood function (centered at the MPV of $\theta_{s i}$ ) results in the following equation.

$$
J_{i}\left(\varphi_{i}, \lambda_{i}, \hat{\theta}_{s i}\right)=L_{s i}\left(\hat{\theta}_{s i}\right)-\varphi_{i}^{T} \hat{\Delta}_{i} \varphi_{i}+\lambda_{i}\left(\varphi_{i}^{T} \varphi_{i}-1\right)
$$

where $L_{s i}\left(\widehat{\theta}_{s i}\right)=$ optimal value of first part, and $\lambda_{i}$ is the Lagrange multiplier that enforces the unit norm of the local mode shape. Minimization of Eq. (8) with respect to $\varphi_{i}$ gives,

$$
\left.\frac{\partial J_{i}\left(\varphi_{i}, \lambda_{i}\right)}{\partial \varphi_{i}}\right|_{\varphi_{i}=\hat{\varphi}_{i}}=-2 \hat{\varphi}_{i}^{T} \hat{\Delta}_{i}+2 \lambda_{i} \hat{\varphi}_{i}^{T}=0
$$

Eq. (9) can be solved as a standard eigenvalue problem which results in the MPV of $\varphi_{i}$. Further, it is seen that the optimal value of $\lambda_{i}$ equals to the optimum $\ell_{i}\left(\hat{\theta}_{s i}\right)$ which corresponds to the maximum eigenvalue of $\widehat{\Delta}_{i}$.

$$
\hat{\lambda}_{i}=\hat{\varphi}_{i}^{T} \hat{\Delta}_{i} \hat{\varphi}_{i}
$$

\section{Analytical solution for mode shape assembly}

\subsection{Proposed solution by two-stage BFFTA}

In this section, an alternative mode shape assembly algorithm is presented. The two-stage Bayesian approach, which is previously applied for single setup problem by Au [19], is adopted to multiple setup case. At first stage of the proposed methodology, the MPV of spectrum variables are obtained by minimizing Eq. (7) with respect to $\theta_{s i}$. At second stage, the constrained negative logarithm likelihood function (centered at the MPV of spectrum parameters) for local mode shape at an individual setup, is obtained by arranging Eq. (8), as below. 


$$
J_{i}\left(\varphi_{i}, \lambda_{i}, \hat{\theta}_{s i}\right)=L_{s i}\left(\hat{\theta}_{s i}\right)-\hat{\lambda}_{i}+\varphi^{T}\left(-\hat{\Delta}_{i}+\hat{\lambda}_{i}\right) \varphi_{i}
$$

Neglecting the constant terms that do not affect the variation of $\varphi_{i}$ leads to the following relation.

$$
J_{i}\left(\varphi_{i}, \lambda_{i}, \hat{\theta}_{s i}\right) \propto \frac{1}{2} \varphi_{i}^{T} H_{\hat{\varphi}_{i}} \varphi_{i}
$$

Here, $H_{\widehat{\varphi}_{i}}=$ Hessian matrix of $J_{i}\left(\varphi_{i}, \lambda_{i}, \widehat{\theta}_{s i}\right)$ with respect to $\varphi_{i}$ (with unit norm) at $\theta_{i}=\widehat{\theta}_{i}$. $H_{\widehat{\varphi}_{i}}$ can be calculated numerically using finite difference method or can be derived analytically. For both procedures, the equality constraints that arises due to the norm of the local mode shape should be considered [4,19,21]. Otherwise, $H_{\widehat{\varphi}_{i}}$ will be a negative definite matrix, and therefore it will inevitably contradict the minimization nature of the MPV of mode shape [4]. $H_{\widehat{\varphi}_{i}}$ can be analytically derived by following the methodology that is proposed by Au and Xie [21] or can be simply obtained by double differentiating of Eq. (8) with respect to $\widehat{\varphi}_{i}$.

$$
H_{\hat{\varphi}_{i}}=\left.\frac{\partial^{2} J_{i}\left(\varphi_{i}, \lambda_{i}, \hat{\theta}_{s i}\right)}{\partial \varphi_{i}^{2}}\right|_{\varphi_{i}=\hat{\varphi}_{i}}=-2 \hat{\Delta}_{i}+2 \hat{\lambda}_{i} I_{N_{i}}
$$

The modal shapes of individual setups are statistically independent [18]. Thus, the objective functions for each setup can be assembled by linear combination, and the resulting objective function for the global mode vector, $\Phi$, under norm constraints can be written as follows by substituting $\varphi_{i}=r_{i}^{-1} \Gamma_{i} \Phi$, where $\Gamma_{i}$ is the selection matrix that extracts the DOFs measured at $i^{\text {th }}$ setup.

$$
J(\Phi, \alpha, \beta, r)=\frac{1}{2} \sum_{i=1}^{N_{s}} r_{i}^{-2} \Phi^{T} \Gamma_{i}^{T} H_{\hat{\varphi}_{i}} \Gamma_{i} \Phi+\sum_{i=1}^{N_{s}} \alpha_{i}\left(\Phi^{T} \Gamma_{i}^{T} \Gamma_{i} \Phi-r_{i}^{2}\right)+\beta\left(1-\Phi^{T} \Phi\right)
$$

Here, $\alpha=\left[\alpha_{1}, \ldots, \alpha_{N s}\right), r=\left[r_{1}, \ldots, r_{N s}\right], \alpha_{i}=$ Lagrange multiplier for the norm constraint of $\left\|\Gamma_{i} \Phi\right\|=r_{i}$, and $\beta=$ Lagrange multiplier that enforces the unit norm for $\Phi$. The Lagrange multiplier $\alpha_{i}$ is obtained at the minimum value of Eq. (14) with respect to $r_{i}$.

$$
\frac{\partial J}{\partial r_{i}}=-r_{i}^{-3} \Phi^{T} \Gamma_{i}^{T} H_{\hat{\varphi}_{i}} \Gamma_{i} \Phi-2 \alpha_{i} r_{i}=0 \Rightarrow \hat{\alpha}_{i}=-\frac{r_{i}^{-4}}{2} \Phi^{T} \Gamma_{i}^{T} H_{\hat{\varphi}_{i}} \Gamma_{i} \Phi
$$

Note that the second order derivative of the objective function with respect to $r_{i}$ is a non-negative value since $H_{\widehat{\varphi}_{i}}$ is semipositive definite [19].

$$
\frac{\partial^{2} J}{\partial r_{i}^{2}}=3 r_{i}^{-4} \Phi^{T} \Gamma_{i}^{T} H_{\hat{\varphi}_{i}} \Gamma_{i} \Phi-2 \hat{\alpha}_{i}=4 r_{i}^{-4} \Phi^{T} \Gamma_{i}^{T} H_{\hat{\varphi}_{i}} \Gamma_{i} \Phi \geq 0
$$

Minimizing Eq. (14) with respect to $\Phi$ leads to

$$
\left.\frac{\partial J}{\partial \Phi}\right|_{\Phi=\hat{\Phi}}=\hat{\Phi}^{T}\left[\sum_{i=1}^{N_{s}} r_{i}^{-2} \Gamma_{i}^{T} H_{\hat{\varphi}_{i}} \Gamma_{i}\right]+\hat{\Phi}^{T}\left[\sum_{i=1}^{N_{s}} 2 \hat{\alpha}_{i} \Gamma_{i}^{T} \Gamma_{i}\right]+2 \beta \hat{\Phi}^{T}=0
$$

Thus, the most probable global mode shape vector is obtained by the solution of the following standard eigenvalue problem.

$$
\hat{A} \Phi=\beta \Phi
$$

in which

$$
\hat{A}=\frac{1}{2} \sum_{i=1}^{N_{s}} \Gamma_{i}^{T}\left(r_{i}^{-2} H_{\hat{\varphi}_{i}}+2 \hat{\alpha}_{i} I_{N i}\right) \Gamma_{i}
$$

\subsection{Alternative solution by Gaussian approximation}

In this section, it is shown that the solution for the global mode shape vector is unique regardless of the implemented assembly methodology. For this purpose, the final solution for the global mode shape in Eq. (18) is obtained by following a similar procedure to the method by Yan and Katafygiotis [20]. Since the problem is globally identifiable, the conditional PDF for $i^{\text {th }}$ local mode shape centered at the MPV for spectrum variables can be estimated by Gaussian approximation. For this purpose, the conditional PDF for global mode shape can be written as below.

$$
p\left(\Phi \mid\left[\varphi_{i} \ldots \varphi_{N_{s}}\right]\right)=\prod_{i=1}^{N_{s}} p\left(\Phi \mid \varphi_{i}\right)
$$


According to the Bayes' theorem, the following equality can be constructed.

$$
p\left(\Phi \mid \varphi_{i}\right)=p\left(\varphi_{i} \mid \Phi\right) p(\Phi) / p\left(\varphi_{i}\right)
$$

where $p\left(\Phi \mid \varphi_{i}\right)=$ posterior conditional pdf for global mode shape $\Phi$ for a given $\varphi_{i}, p\left(\varphi_{i} \mid \Phi\right)=$ likelihood function of local mode shape $\varphi_{i}$ that is conditional to $\Phi, p(\Phi)$ and $p\left(\varphi_{i}\right)$ are prior distributions $\Phi$ and $\varphi_{i}$, respectively. Here, $p\left(\varphi_{i}\right)$ corresponds to a normalizing constant since it does not depend on $\Phi$. In addition, $p(\Phi)$ does not depend on the measured data and should be defined by the user. In case of large amount of data, the variation in $p\left(\Phi \mid \varphi_{i}\right)$ is significantly larger than the variation in $p(\Phi)$ [19]. Therefore, the contribution of $p(\Phi)$ can be neglected, and $p\left(\Phi \mid \varphi_{i}\right)$ can be assumed to be proportional to $p\left(\varphi_{i} \mid \Phi\right)$. Thus, $p\left(\Phi \mid \varphi_{i}\right)$ can be well estimated by Gaussian approximation around the MPV of local mode shape.

$$
p\left(\Phi \mid \varphi_{i}\right) \propto p\left(\varphi_{i} \mid \Phi\right)=\left|H_{\hat{\varphi}_{i}}^{-1}\right|(2 \pi)^{-N / 2} \exp \left\{-\frac{1}{2}\left(r_{i}^{-1} \Gamma_{i} \Phi-\hat{\varphi}_{i}\right)^{T} H_{\hat{\varphi}_{i}}\left(r_{i}^{-1} \Gamma_{i} \Phi-\hat{\varphi}_{i}\right)\right\}
$$

Here, $H_{\widehat{\varphi}_{i}}$ is used instead of the inverse of the posterior covariance matrix. Thus, the negative logarithm likelihood function of $p\left(\Phi \mid \varphi_{i}\right)$ is written as;

$$
\begin{aligned}
& L_{i}\left(\Phi, r_{i}\right)=-\ln \left[p\left(\varphi_{i} \mid \Phi\right)\right] \\
& L_{i}\left(\Phi, r_{i}\right)=\frac{1}{2} N \ln \pi+\frac{1}{2} \ln \left|H_{\hat{\varphi}_{i}}^{-1}\right|+\frac{1}{2}\left(r_{i}^{-1} \Gamma_{i} \Phi-\hat{\varphi}_{i}\right)^{T} H_{\hat{\varphi}_{i}}\left(r_{i}^{-1} \Gamma_{i} \Phi-\hat{\varphi}_{i}\right)
\end{aligned}
$$

Applying the norm constraint for the local mode shape, the objective function for the $i^{\text {th }}$ setup is obtained as;

$$
J_{i}\left(\Phi, \alpha_{i}, r_{i}\right)=L_{i}\left(\Phi, r_{i}\right)+\alpha_{i}\left(\Phi^{T} \Gamma_{i}^{T} \Gamma_{i} \Phi-r_{i}^{2}\right)
$$

Taking the first order derivative of Eq. (24) with respect to $r_{i}$ gives

$$
\frac{\partial J_{i}}{\partial r_{i}}=r_{i}^{-2} \Phi^{T} \Gamma_{i}^{T} H_{\hat{\varphi}_{i}} \hat{\varphi}_{i}-r_{i}^{-3} \Phi^{T} \Gamma_{i}^{T} H_{\hat{\varphi}_{i}} \Gamma_{i} \Phi-2 \alpha_{i} r_{i}=0
$$

Thus, the optimal value of $\alpha_{i}$ is obtained as

$$
\hat{\alpha}_{i}=-\frac{r_{i}^{-4}}{2} \Phi^{T} \Gamma_{i}^{T} H_{\hat{\varphi}_{i}} \Gamma_{i} \Phi+\frac{r_{i}^{-3}}{2} \Phi^{T} \Gamma_{i}^{T} H_{\hat{\varphi}_{i}} \hat{\varphi}_{i}
$$

Finally, the objective function for the global mode vector under norm constraints is written as

$$
J(\Phi, \alpha, \beta, r)=\frac{1}{2} \sum_{i=1}^{N_{s}}\left(r_{i}^{-1} \Gamma_{i} \Phi-\hat{\varphi}_{i}\right)^{T} H_{\hat{\varphi}_{i}}\left(r_{i}^{-1} \Gamma_{i} \Phi-\hat{\varphi}_{i}\right)+\sum_{i=1}^{N_{s}} \alpha_{i}\left(\Phi^{T} \Gamma_{i}^{T} \Gamma_{i} \Phi-r_{i}^{2}\right)+\beta\left(1-\Phi^{T} \Phi\right)
$$

Minimizing Eq. (27) with respect to $\Phi$ leads to the following constrained eigenvalue problem.

$$
\begin{aligned}
& \mathrm{A} \Phi+\mathrm{b}=\beta \Phi \\
& \mathrm{A}=\frac{1}{2} \sum_{i=1}^{N_{s}} \Gamma_{i}^{T}\left(r_{i}^{-2} H_{\hat{\varphi}_{i}}+2 \hat{\alpha}_{i} I_{N i}\right) \Gamma_{i} ; \mathrm{b}=-\frac{1}{2} \sum_{i=1}^{N_{s}} r_{i}^{-1} \Gamma_{i}^{T} \hat{H}_{\varphi_{i}} \hat{\varphi}_{i}
\end{aligned}
$$

The obtained constrained eigenvalue problem is similar to the result by Yan and Katafygiotis [20]. For this alternative solution, however, Eq. (28) is reduced into a standard eigenvalue problem. For this purpose, the components of $H_{\widehat{\varphi}_{i}}$ can be expressed in terms of the eigenvalue decomposition of its two terms.

$$
\begin{aligned}
& H_{\hat{\varphi}_{i}}=-2 \hat{\Delta}_{i}+2 \hat{\lambda}_{i} I_{N_{i}}=U \Sigma U^{T}+2 \hat{\lambda}_{i} U U^{T} \\
& U=\left[\begin{array}{llll}
u_{1}=\hat{\varphi}_{i} & u_{2} & \ldots & u_{N_{i}}
\end{array}\right] \\
& \Sigma=\operatorname{diag}\left[\sigma_{1}=-2 \hat{\lambda}_{i} \quad \sigma_{2} \quad \ldots \quad \sigma_{N_{i}}\right] \\
& \left|\sigma_{1}\right|=\max \left(\left|\sigma_{1}\right|, \ldots,\left|\sigma_{N_{i}}\right|\right) ; \quad U U^{T}=I_{N_{i}}
\end{aligned}
$$

Then, the equivalent eigenvalue decomposition of $H_{\widehat{\varphi}_{i}}$ is written by combining the decomposed terms in Eq. (29).

$$
\begin{aligned}
& H_{\hat{\varphi}_{i}}=U \bar{\Sigma} U^{T} \\
& U=\left[\begin{array}{llll}
\hat{\varphi}_{i} & u_{2} & \ldots & u_{N_{i}}
\end{array}\right] \\
& \bar{\Sigma}=\operatorname{diag}\left[\begin{array}{llll}
0 & \left(\sigma_{2}+2 \hat{\lambda}_{i}\right) & \ldots & \left(\sigma_{N_{i}}+2 \hat{\lambda}_{i}\right.
\end{array}\right]
\end{aligned}
$$

In Eq. (30), $H_{\widehat{\varphi}_{i}}$ is semi-positive definite and, its eigenvalue that corresponds to $\widehat{\varphi}_{i}$ is equal to zero. Therefore, the vector of $b$ will be a zero vector.

$$
H_{\hat{\varphi}_{i}} \hat{\varphi}_{i}=0_{N_{i \times 1}} \Rightarrow b=0_{N_{\times 1}}
$$

Thus, Eq. (28) leads to the same standard eigenvalue problem that is given in Eq. (18). 


\subsection{Posterior uncertainty for global mode shape}

Posterior uncertainty for the local mode shape vectors can be calculated in terms of their Expected Modal Assurance Criterion (EMAC) for each setup [3,22]. The assembled global mode shape vector has identification uncertainty, as well. To define this uncertainty, the posterior covariance matrix for global mode shape should be obtained first. The posterior covariance matrix depends on the defined constraints. Taking the second order derivatives of the objective function that is given in Eq. (14) for the global mode shape vector gives the Hessian at $\Phi=\widehat{\Phi}$.

The posterior covariance matrix, $C_{\widehat{\Phi}}$ can be obtained as the inverse of the Hessian matrix, $H_{\widehat{\Phi}}$. However, this procedure inevitably causes numerical errors since $H_{\widehat{\varphi}}$ is a semi-positive definite matrix. The pseudo inverse of $H_{\widehat{\Phi}}$ can be used to calculate $C_{\widehat{\Phi}}$ [22]. For this purpose, the singular value decomposition of $H_{\widehat{\Phi}}$ can be written as;

$$
H_{\hat{\Phi}}=0 \times \Phi \Phi^{T}+\sum_{j=2}^{N} \delta_{\varphi \cdot j}^{2} \rho_{j} \rho_{j}^{T}
$$

Thus, the posterior covariance matrix is written as follows by neglecting the zero eigenvalue,

$$
C_{\hat{\Phi}}=H_{\hat{\Phi}}^{+}=\sum_{j=2}^{N} \delta_{\hat{\Phi}, j}^{-2} \rho_{j} \rho_{j}^{T}
$$

where "+" denotes the pseudo-inverse. The uncertainty of mode shape with covariance matrix, $C_{\widehat{\Phi}}$, can be defined in vectoral form as the difference between the uncertain and most probable global mode shape [19].

$$
\Delta \Phi=\Phi-\widehat{\Phi}=\sum_{j=2}^{N} z_{j} \delta_{\widehat{\Phi} . j}^{-1} \rho_{j}
$$

Here, $z_{j}=$ independent and identically distributed Gaussian number. Thus, EMAC between uncertain and most probable mode shapes are obtained as below.

$$
\begin{gathered}
\Phi=\left(\widehat{\Phi}+\sum_{j=2}^{N} z_{j} \delta_{\Phi, j}^{-1} \rho_{j}\right) \\
E M A C=\frac{\Phi^{\mathrm{T}} \widehat{\Phi}}{\|\Phi\|\|\widehat{\Phi}\|}=\frac{1}{\sqrt{1+\sum_{j=2}^{N} z_{j}^{-2} \delta_{\Phi, j}^{-2}}}
\end{gathered}
$$

Zhang and $\mathrm{Au}[22]$ states that EMAC asymptotically leads to the following expression as $N \rightarrow \infty$, and $\delta_{\widehat{\Phi} ; j}{ }^{-2} \rightarrow 0$.

$$
E M A C=\left(1+\sum_{j=2}^{N} \delta_{\widehat{\Phi}, j}^{-2}\right)^{-1}
$$

\section{Estimation of the weights for individual setups}

In the assembly procedure, local mode shapes that are identified in the individual setups have a contribution to the global mode shape. The Global Least Squares approach assumes that all setups are equally weighted, and their contribution is uniform. Bayesian methods $[19,20]$, however, considers the weight of each setup incorporating the identification quality. In this context, Au [19] presents the following asymptotic weighting factor in case of large signal to noise ratio.

$$
w_{i} \propto \frac{r_{i}^{-2} \lambda_{i o}}{S_{e i}}
$$

in which $\lambda_{i o}$ is the largest eigenvalue of $\sum_{k} \operatorname{Re}\left(F_{k i} F_{k i}^{*}\right)$.

In case of lower signal-to-noise ratio, the weighting of each setup can also be calculated as depending on the data quality. For this purpose, the local Hessian matrix, $H_{\widehat{\varphi}_{i}}$, can be rewritten as follows by neglecting the zero-eigenvalue term along the local mode shape direction.

$$
H_{\hat{\varphi}_{i}} \approx \frac{\delta_{\hat{\varphi}_{i}}}{\left(N_{i}-1\right)}\left[I_{N_{i}}-\hat{\varphi}_{i} \hat{\varphi}_{i}^{T}\right]
$$

where $\delta_{\widehat{\varphi}_{i}}=$ sum of all eigenvalues of $H_{\widehat{\varphi}_{i}}$. By using Eq. (38), the optimal value of the Lagrange multiplier, $\alpha_{i}$, can be arranged as,

$$
\hat{\alpha}_{i}=\frac{r_{i}^{-4} \delta_{\hat{\varphi}_{i}}}{\left(N_{i}-1\right)}\left[r_{i}^{-2}-\Phi^{T} \Gamma_{i}^{T} \hat{\varphi}_{i} \hat{\varphi}_{i}^{T} \Gamma_{i} \Phi\right]
$$


Substituting Eqs. (38) and (39) into Eq. (19) yields,

$$
\hat{A}=\sum_{i=1}^{N_{s}} \frac{r_{i}^{-2} \delta_{\hat{\varphi}_{i}}}{\left(N_{i}-1\right)} \Gamma_{i}^{T}\left[\bar{\alpha}_{i} I_{N_{i}}-\hat{\varphi}_{i} \hat{\varphi}_{i}^{T}\right] \Gamma_{i}
$$

where

$$
\bar{\alpha}_{i}=r_{i}^{-2} \Phi^{T} \Gamma_{i}^{T} \hat{\varphi}_{i} \hat{\varphi}_{i}^{T} \Gamma_{i} \Phi
$$

Thus, the weight of the $i^{\text {th }}$ setup in the optimal global mode shape can be estimated by the following formula.

$$
w_{i}=\frac{r_{i}^{-2} \delta_{\hat{\varphi}_{i}}}{2\left(N_{i}-1\right)}
$$

\section{Summary of procedure}

The proposed modified solution assembles mode shapes by using the probability distributions for local mode shapes centered at the local spectrum variables. First, the spectrum variables at $i^{\text {th }}$ setup should be determined, and the Hessian matrix for each local modal shape should be obtained. Second, the global mode shape vector is assembled by the scaled local Hessian matrices and possible discrepancy.

In the proposed procedure, the possible modes should be detected from the singular value (SV) or power spectral density (PSD) spectrum of the measured data by peak-picking. At the next step, a proper bandwidth should be selected. An automated mode detection and bandwidth selection for BAYOMA are not available in the literature. For this reason, the possible mode and bandwidth selection inevitably becomes an intuitional procedure. In this study, the bandwidths of possible modes are selected so that it covers the bell curve in the SV spectrum.

The local spectrum parameters cannot be directly obtained by the minimization of Eq. (7) since it is a multivariate non-linear function. Instead, an iterative approach should be implemented to find the global minimum with respect to the spectrum parameters. In this procedure, selection of a proper initial guess for each parameter will significantly decrease the computational effort and time. For this purpose, the initial guess for $f_{i}$ can be set to the peak value in the selected bandwidth, and the initial value of $\xi_{i}$ can be set to $1 \%$ [3]. In addition, the initial guess for $\bar{S}_{i}$ and $S_{e i}$ can be calculated by making use of the asymptotic behavior of signal-to-noise ratio for its larger values [3]. Here the signal-to-noise ratio is defined as below.

$$
\gamma_{k i}=\frac{\bar{S}_{i} D_{k i}}{S_{e i}}
$$

In case of a significantly large signal-to-noise ratio, matrix of $\Delta_{i}$ can be rewritten by using the first order power series expansion of the following term [3].

$$
\left(1+\frac{S_{e i}}{\bar{S}_{i} D_{k i}}\right)^{-1} \approx\left(1-\frac{S_{e i}}{\bar{S}_{i} D_{k i}}\right)
$$

Then,

$$
\Delta_{i}=\sum_{k} S_{e i}^{-1}\left(1+\frac{S_{e i}}{\bar{S}_{i} D_{k i}}\right)^{-1} F_{k i} F_{k i}^{*} \approx S_{e i}^{-1} \sum_{k} F_{k i} F_{k i}^{*}-\sum_{k} \frac{F_{k i} F_{k i}^{*}}{\bar{S}_{i} D_{k i}}
$$

By making use of Eqs. (44) and (45), the likelihood function given in Eq. (7) can be arranged as follows

$$
L\left(\theta_{s i}\right) \approx \underbrace{\sum_{k} \ln D_{k}}_{\text {Sensitive to } f_{i} \text { and } \xi_{i}}+\underbrace{\left[N_{f_{i}}\left(N_{i}-1\right) \ln S_{e_{i}}+S_{e}^{-1}\left(\kappa_{i}-\lambda_{i o}\right)\right]}_{\text {Sensitive to } S_{e i}}+\underbrace{\left[N_{f i} \ln \bar{S}_{i}+\bar{S}_{i}^{-1} \sum_{k} \lambda_{k i} D_{k i}^{-1}\right]}_{\text {Sensitive to } f_{i}, \xi_{i} \text { and } \bar{S}_{i}}
$$

where $\lambda_{i o}=$ maximum eigenvalue of $\sum_{k} \operatorname{Re}\left(F_{k i} F_{k i}^{*}\right)$, and $\lambda_{k i}=$ maximum eigenvalue of $\operatorname{Re}\left(F_{k i} F_{k i}^{*}\right)$. Minimizing Eq. (46) with respect to $\bar{S}_{i}$ and $S_{e i}$ gives their optimal values as below.

$$
S_{e i} \cong \frac{\kappa_{i}-\lambda_{i o}}{\left(N_{i}-1\right) N_{f i}} ; \quad \bar{S}_{i} \cong \sum_{k} \lambda_{k i} D_{k i}^{-1} ;
$$

In the calculation of the global mode shape, again an iterative procedure should be applied since Eq. (19) depends on $r_{i}$ and $\widehat{\alpha}_{i}$, which are related to the global mode shape. In this iterative procedure, an initial guess for the global mode shape can be selected by assuming that the local part of $\widehat{\Phi}$ is well matched with the identified local mode shape $\hat{\varphi}_{i}$ (zero discrepancy). This assumption removes the Lagrange multiplier, $\widehat{\alpha}_{i}$ since $\widehat{\varphi}_{i}$ is the null vector of $H_{\widehat{\varphi}_{i}}$. Thus, the resulting equation only depends on $r_{i}, \Gamma_{\mathrm{i}}$ and $H_{\widehat{\varphi}_{i}}$. 


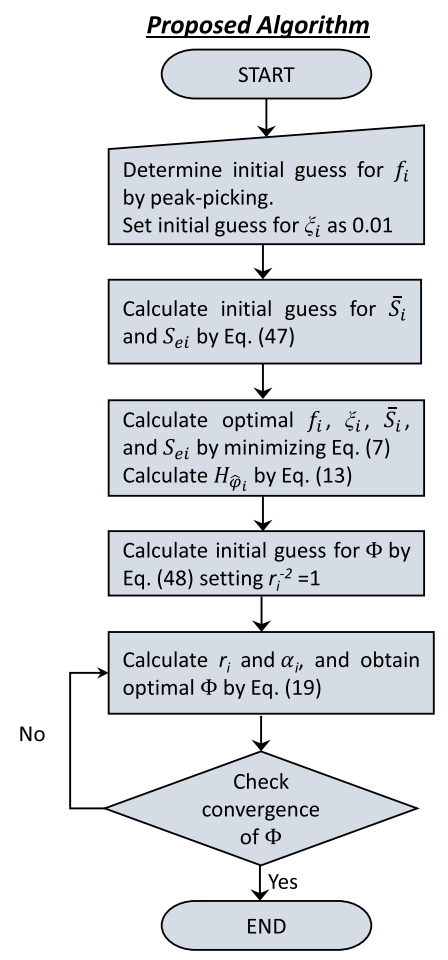

Fig. 1. Flow chart for the proposed algorithm.

Table 1

Multiple setup configuration.

\begin{tabular}{ll}
\hline Setup Number & Measured DOFs \\
\hline 1 & $10,9,8,7$ \\
2 & $8,7,6,5$ \\
3 & $6,5,4,3$ \\
4 & $4,3,2,1$ \\
\hline
\end{tabular}

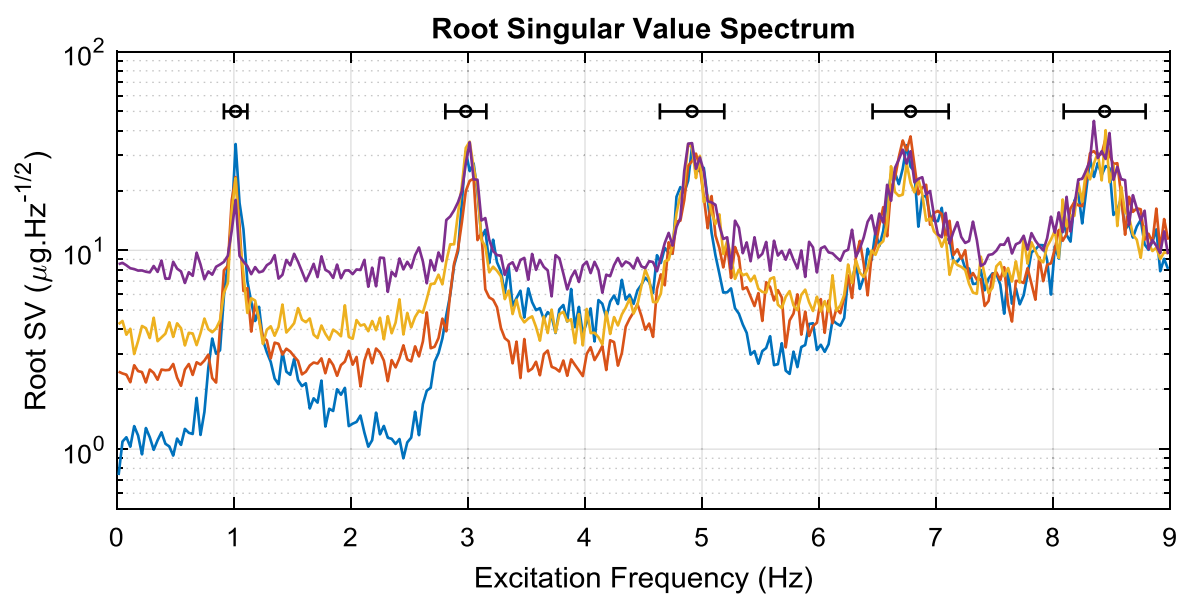

Fig. 2. Maximum root-singular value spectra of the measured setups. 
Table 2

Average MPVs with posterior coefficient of variations (c.o.v.) for $f_{i}$ and $\xi_{i}$.

\begin{tabular}{|c|c|c|c|c|c|c|}
\hline \multirow[t]{2}{*}{ Mode \# } & \multicolumn{3}{|c|}{ Frequency $(\mathrm{Hz})$} & \multicolumn{3}{|c|}{ Damping ratio (\%) } \\
\hline & Actual & MPV & c.o.v. (\%) & Actual & MPV & c.o.v. (\%) \\
\hline 1 & 1.0092 & 1.0134 & 0.2759 & 1.50 & 1.4835 & 32.5827 \\
\hline 2 & 3.0051 & 3.0083 & 0.3121 & 1.50 & 1.4623 & 41.4159 \\
\hline 3 & 4.9338 & 4.9312 & 0.2954 & 1.50 & 1.5416 & 35.6324 \\
\hline 4 & 6.7524 & 6.7495 & 0.3116 & 1.50 & 1.5233 & 40.2148 \\
\hline 5 & 8.4201 & 8.4266 & 0.4482 & 1.50 & 1.5894 & 36.8562 \\
\hline
\end{tabular}

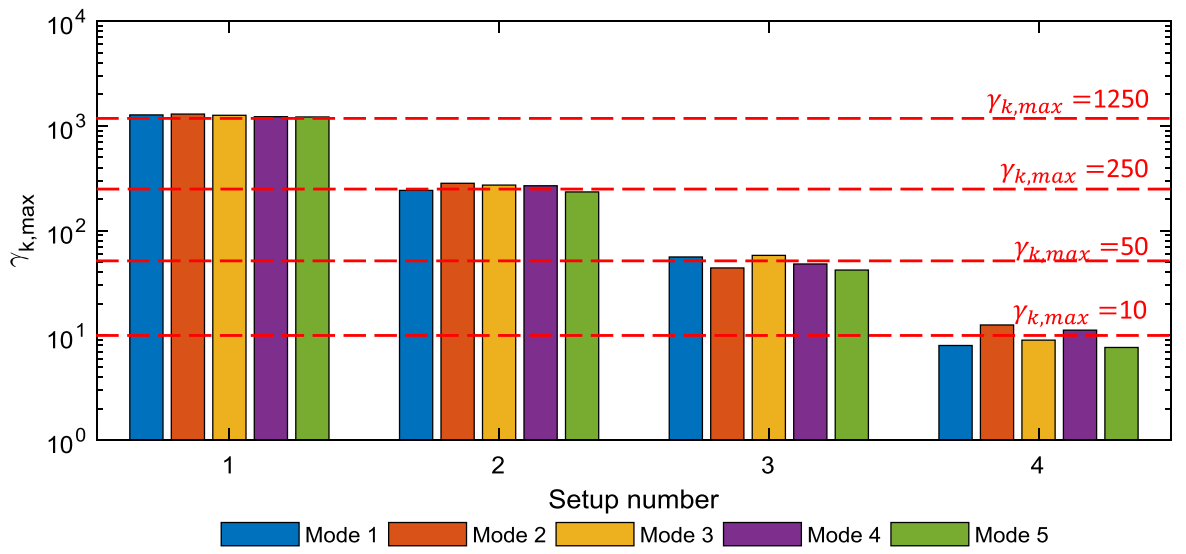

Fig. 3. Identified modal signal-to-noise ratios.

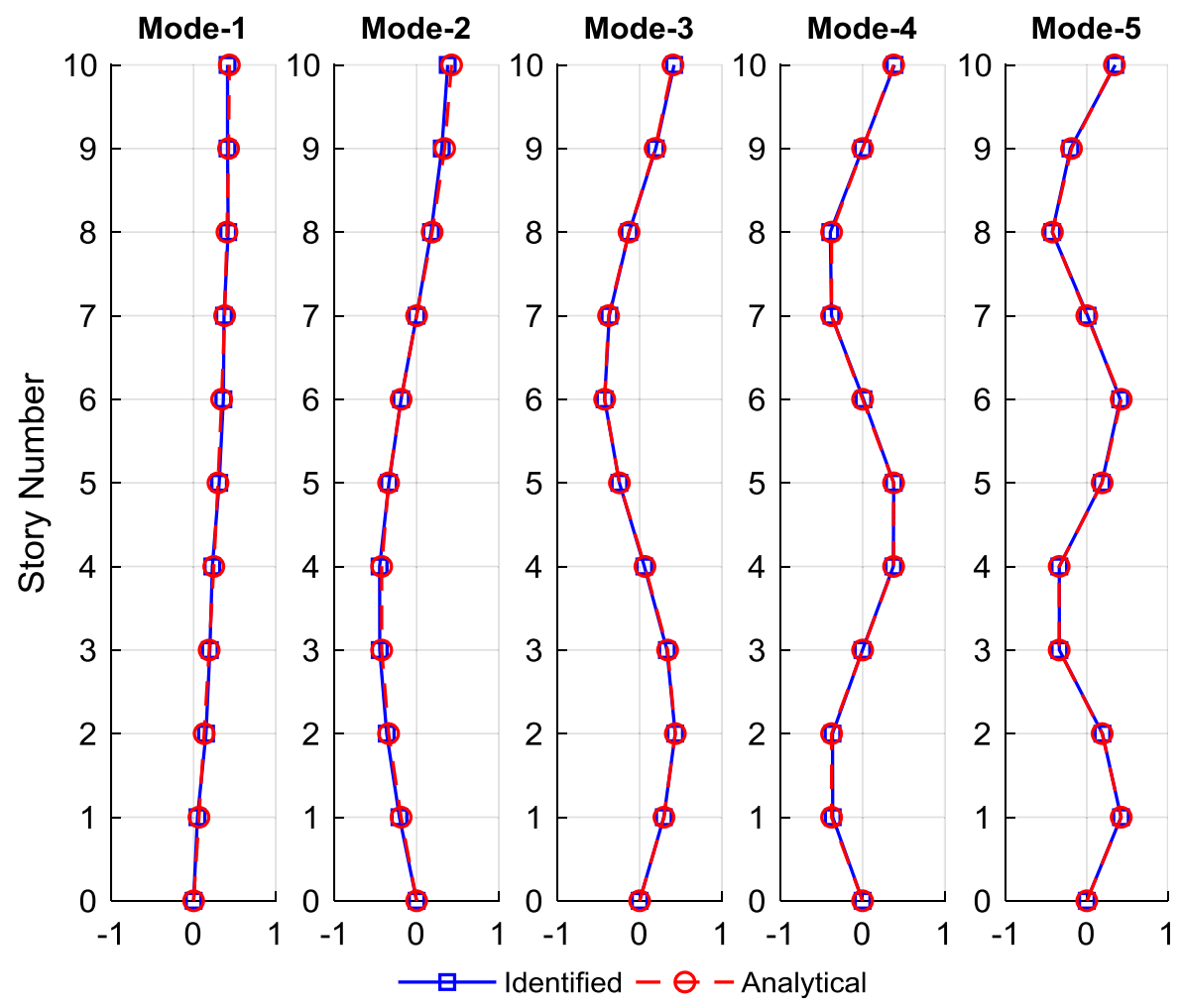

Fig. 4. Identified global mode shapes for the first five modes. 
Measurement

Direction
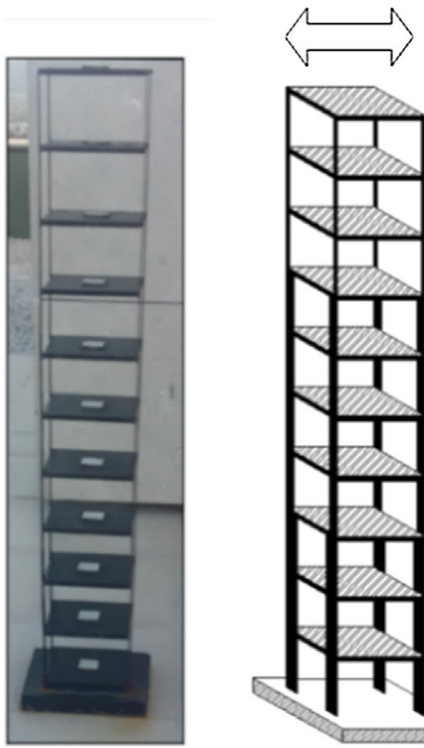

Lumped mass model
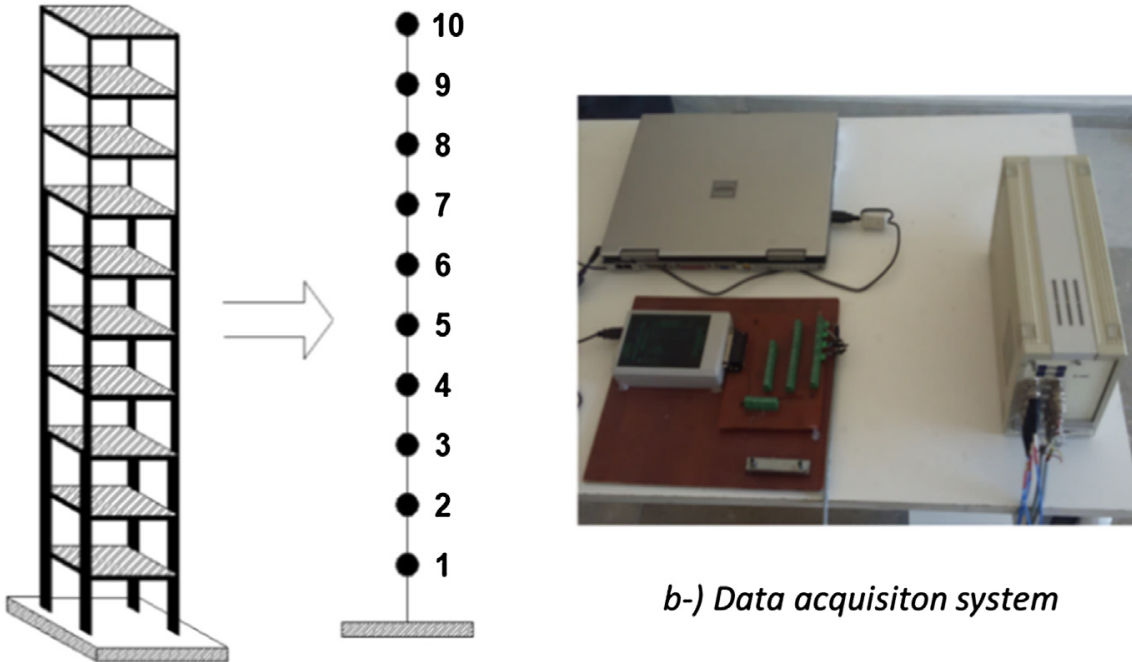

b-) Data acquisiton system

\section{a-) Schematical view of laboratory shear frame}

Fig. 5. Ten story shear frame structure and data acquisition system.

Table 3

Setup configurations for Case-I and Case-II.

\begin{tabular}{lll}
\hline \multirow{2}{*}{ Setup Number } & \multicolumn{2}{l}{ Measured DOFs } \\
\cline { 2 - 3 } & Case-I & Case-II \\
\hline 1 & $1,2,3,10$ & $1,2,3,4$ \\
2 & $1,4,5,10$ & $3,4,5,6$ \\
3 & $1,6,7,10$ & $5,6,7,8$ \\
4 & $1,8,9,10$ & $7,8,9,10$ \\
\hline
\end{tabular}
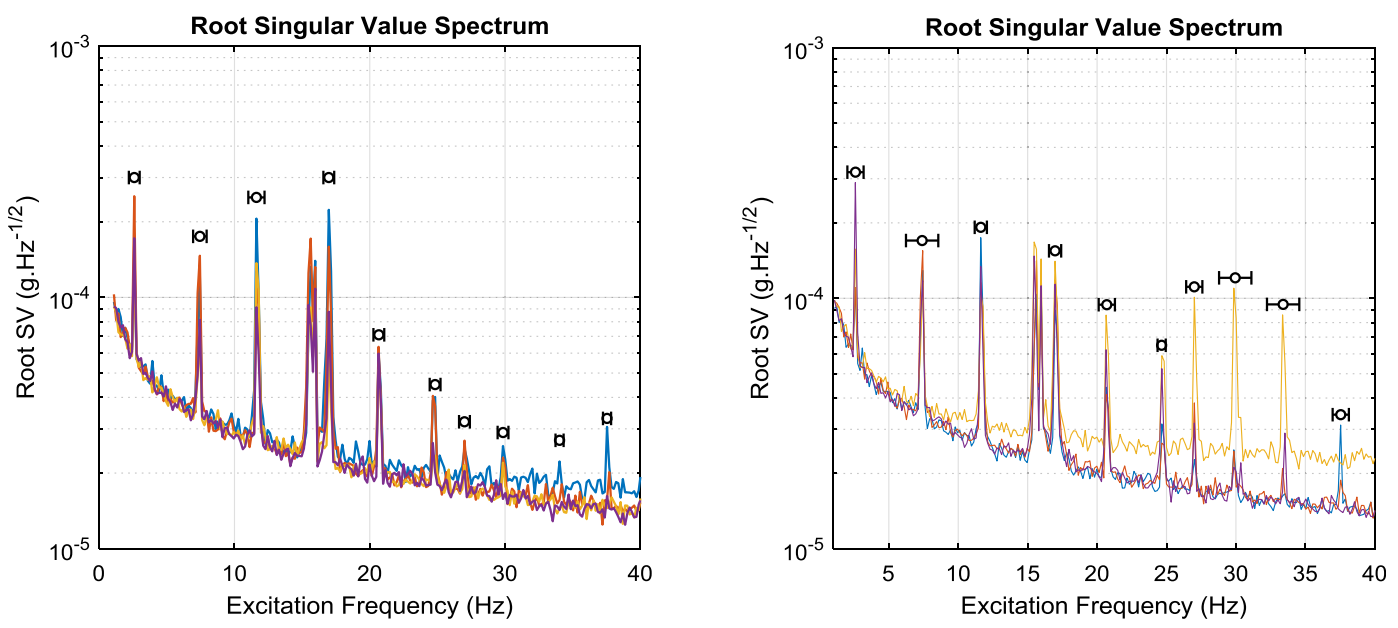

a-) Case-I (Setups 1 to 4)

b-) Case-II (Setups 1 to 4 )

Fig. 6. Maximum root-singular value spectra 


$$
r_{i}^{-1} \Gamma_{i} \Phi=\hat{\varphi}_{i} \quad \Rightarrow \quad \hat{\alpha}_{i}=\hat{\varphi}_{i}^{T} H_{\hat{\varphi}_{i}} \Gamma_{i} \hat{\varphi}_{i}=0 \Rightarrow \hat{A} \approx \sum_{i=1}^{N_{s}} r_{i}^{-2} \Gamma_{i}^{T} H_{\hat{\varphi}_{i}} \Gamma_{i}
$$

In Eq. (48), the Hessian matrix, $H_{\widehat{\varphi}_{i}}$, at each setup is scaled by $r_{i}^{-2}$ and a direct solution is still impossible since $r_{i}$ is initially unknown. To obtain a direct solution for initial guess of $\Phi$, the contribution of $r_{i}^{-2}$ can be assumed to be uniform for each setup (by setting $r_{i}=1$ ). Thus, the initial guess for the global mode shape vector can be taken as the eigenvector of $\sum_{i=1}^{N_{s}} \Gamma_{i}^{T} H_{\widehat{\varphi}_{i}} \Gamma_{i}$ that corresponds to the minimum eigenvalue.

The overall procedure of the proposed algorithm is presented in Fig. 1. The main difference from Au [19] resides in;

- the application of two-stage BFFTA to multiple setups,

- weighting of each setup by the Hessian matrix for local mode shapes

- the calculation of initial guess, and

\section{Table 4}

Average MPVs and representative statistics for $f_{i}$, and $\xi_{i}$.

\begin{tabular}{|c|c|c|c|c|c|c|c|c|c|c|}
\hline \multirow[t]{3}{*}{ Mode \# } & \multicolumn{2}{|c|}{ Reference } & \multicolumn{4}{|l|}{ Case-I } & \multicolumn{4}{|l|}{ Case-II } \\
\hline & \multirow[t]{2}{*}{$f(\mathrm{~Hz})}$. & \multirow[t]{2}{*}{$\xi(\%)$} & \multicolumn{2}{|l|}{$f$} & \multicolumn{2}{|l|}{$\xi$} & \multicolumn{2}{|l|}{$f$} & \multicolumn{2}{|l|}{$\xi$} \\
\hline & & & MPV (Hz.) & c.o.v. (\%) & MPV (Hz.) & c.o.v. (\%) & MPV (Hz.) & c.o.v. (\%) & MPV (Hz.) & c.o.v. (\%) \\
\hline 1 & 2.59 & 0.34 & 2.62 & 0.23 & 0.23 & 26.77 & 2.62 & 0.30 & 0.23 & 14.78 \\
\hline 2 & 7.32 & 0.26 & 7.38 & 0.10 & 0.22 & 44.30 & 7.37 & 0.10 & 0.22 & 11.82 \\
\hline 3 & 11.65 & 0.23 & 11.71 & 0.13 & 0.12 & 19.72 & 11.70 & 0.14 & 0.11 & 25.34 \\
\hline 4 & 16.96 & 0.16 & 17.03 & 0.07 & 0.12 & 46.93 & 17.03 & 0.08 & 0.14 & 24.66 \\
\hline 5 & 20.65 & 0.14 & 20.72 & 0.10 & 0.15 & 30.62 & 20.72 & 0.07 & 0.14 & 26.09 \\
\hline 6 & 24.69 & 0.16 & 24.75 & 0.22 & 0.16 & 38.27 & 24.75 & 0.69 & 0.14 & 61.66 \\
\hline 7 & 26.94 & 0.18 & 27.07 & 0.36 & 0.15 & 55.43 & 27.03 & 0.12 & 0.14 & 36.43 \\
\hline 8 & 29.85 & 0.16 & 29.95 & 0.32 & 0.18 & 33.86 & 29.92 & 0.08 & 0.15 & 22.31 \\
\hline 9 & 33.19 & 0.16 & 33.23 & 0.85 & 0.13 & 47.23 & 33.35 & 0.22 & 0.16 & 21.59 \\
\hline 10 & 37.47 & 0.18 & 37.53 & 0.34 & 0.17 & 28.18 & 37.52 & 0.30 & 0.15 & 34.86 \\
\hline
\end{tabular}
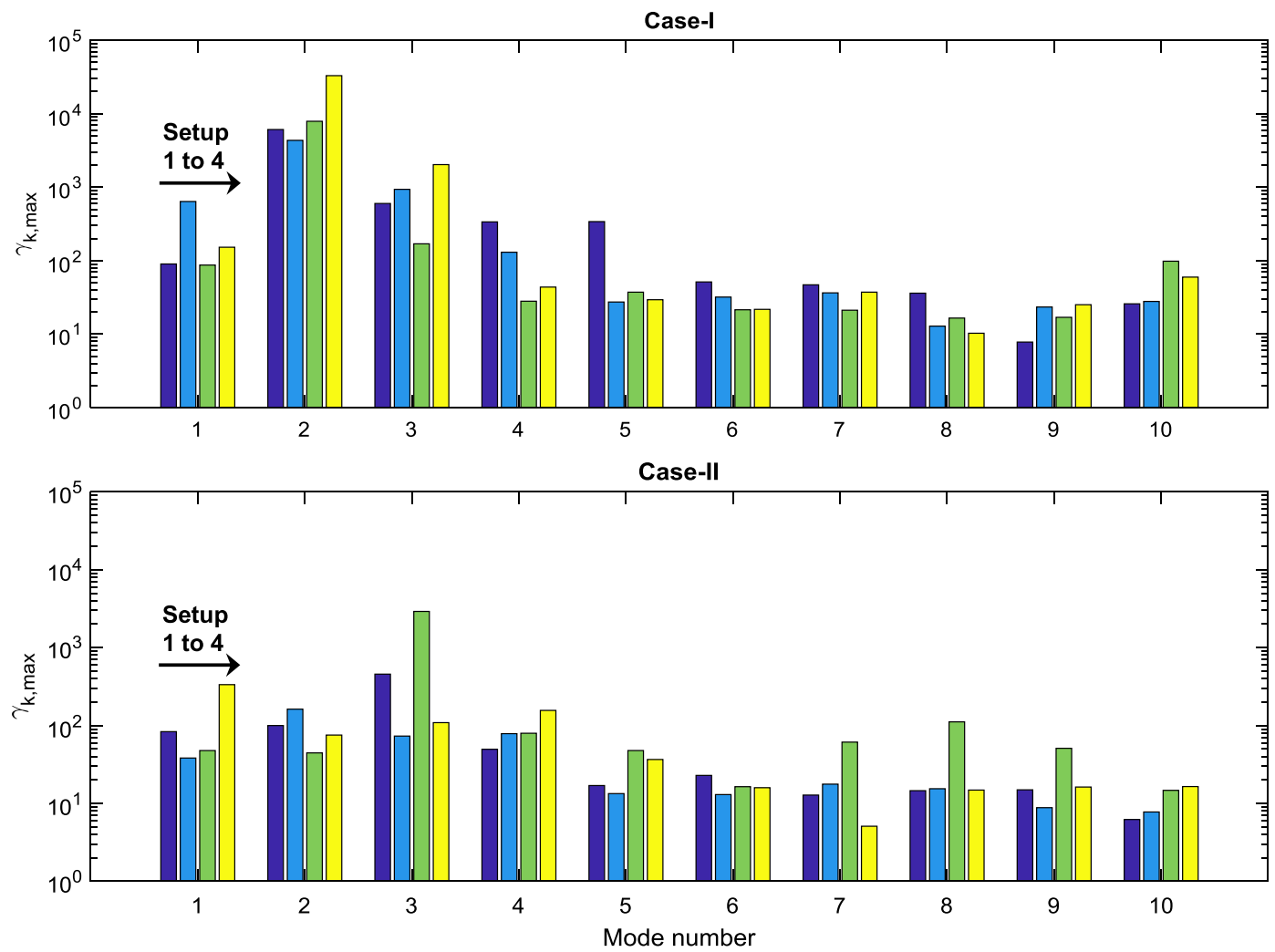

Fig. 7. Variations in the estimated signal-to-noise ratios. 
- the consideration of possible discrepancy between the local part of the global mode shape and the identified local mode shape.

The discrepancy is characterized by defining two different norm constraints for local mode shape. First one, $\hat{\lambda}_{i}$, enforces the unit norm for $\varphi_{i}$, and the second one, $\widehat{\alpha}_{i}$, enforces that $r_{i}=\left\|\Gamma_{i} \Phi\right\|$. Here, the calculation of optimal $\varphi_{i}$ is unnecessary. As the initial guess is close to the optimal value, the convergence speed in the iteration phase is expected to be increased.

\section{Numerical and experimental analysis}

\subsection{Numerical analysis}

A finite element model of a ten-story shear frame is generated with the inter-story stiffness, and story mass as $450 \mathrm{kN} / \mathrm{m}$ and $250 \mathrm{~kg}$, respectively. Modal damping ratio is considered as $1.50 \%$ for all modes. Each floor is subjected to independent and identically distributed (i.i.d.) Gaussian white noise excitation that is generated with $250 \mathrm{~Hz}$ sampling frequency. The generated forcing functions are arranged so that the one-sided PSD of scaled modal excitation is being $\bar{S}_{i}=1 \mu \mathrm{g}^{2} / \mathrm{Hz}$ for all modes and setups. The acceleration response of the structure is divided to four measurement setups which are presented in Table 1. To see the effect of different noise levels on the identification quality, each acceleration response is contaminated by Gaussian white noise. Thereby, the maximum modal signal to noise ratios, $\gamma_{\text {ki.max }}=\bar{S}_{i} /\left(4 \xi_{i}^{2} S_{e i}\right)$, are arranged as 1250,250 , 50 and 10 for setups $1-4$, respectively.

Maximum singular value (SV) spectra of the acceleration responses obtained from the measurement setups is presented in Fig. 2. Possible modes are detected at 1, 3.00, 4.90, 6.75 and $8.42 \mathrm{~Hz}$, for the first five modes respectively. Selected bandwidths for the detected modes are also indicated in Fig. 2. Average values of the identified frequencies and damping ratios,
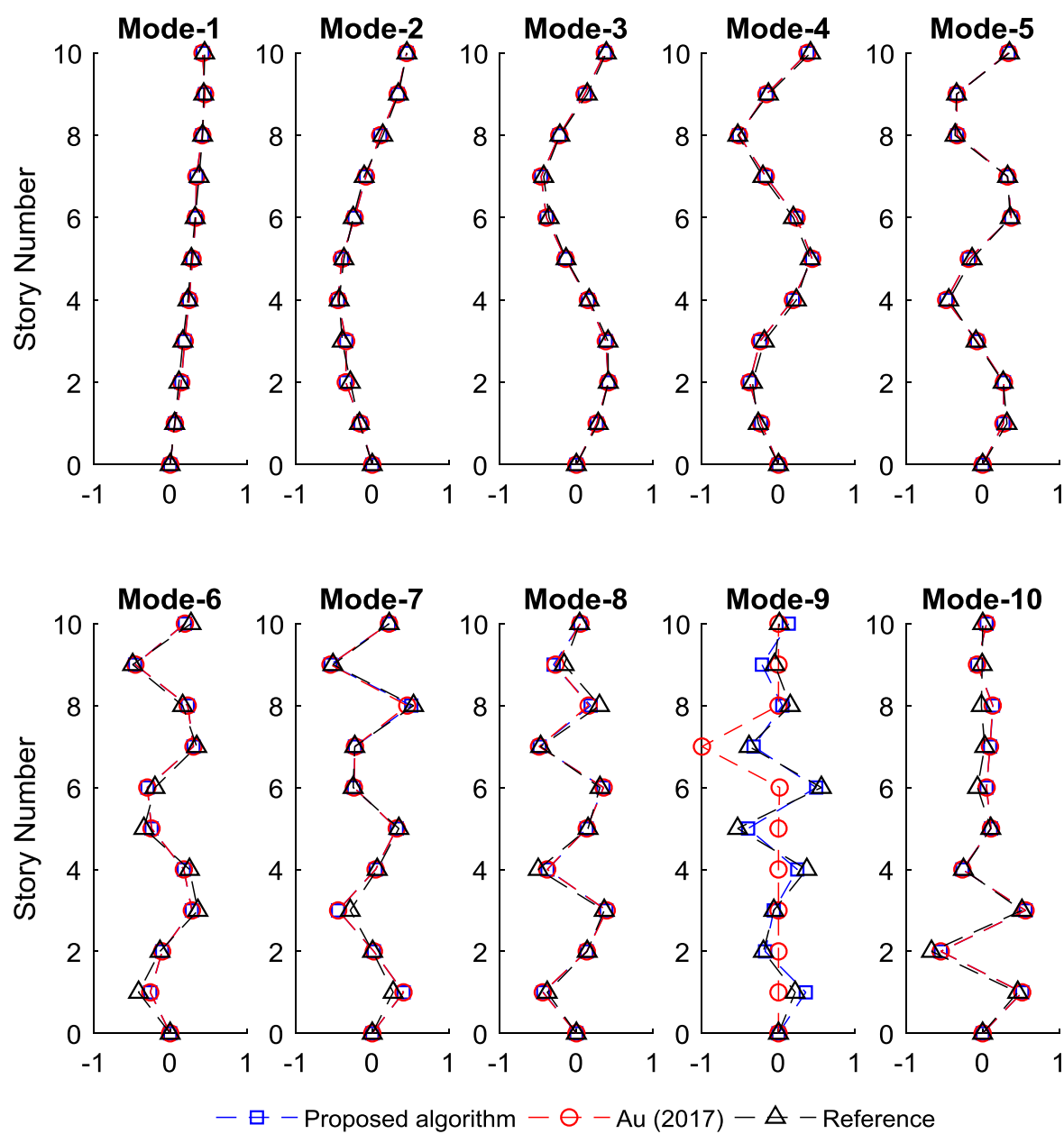

— $\boxminus-$ Proposed algorithm $-\ominus-\mathrm{Au}(2017)-\Delta-$ Reference

Fig. 8. Assembled mode shapes for Case-I. 
and their posterior uncertainties in terms of coefficient of variation (c.o.v.) are presented in Table 2. Here, the coefficients of variation for natural frequencies and damping ratios among all setups include representative statistics. Representative statistics is defined as the combination of setup-to-setup variability and identification uncertainty by Zhang and Au [23]. According to the results, it is observed that the identified frequencies and damping ratios well match with the analytical values. The posterior c.o.v. values are less than $1 \%$ for identified frequencies. For damping ratios, the posterior c.o.v. values increase up to $40 \%$. This observation is compatible with the BFFTA applications in the literature in which the identified damping ratios have relatively larger uncertainties when compared to the identified frequencies $[3,19,23]$. In addition, the variability of the noise levels among different setups is also considered to increase the uncertainty levels. Identified signal-to-noise ratios are pre-
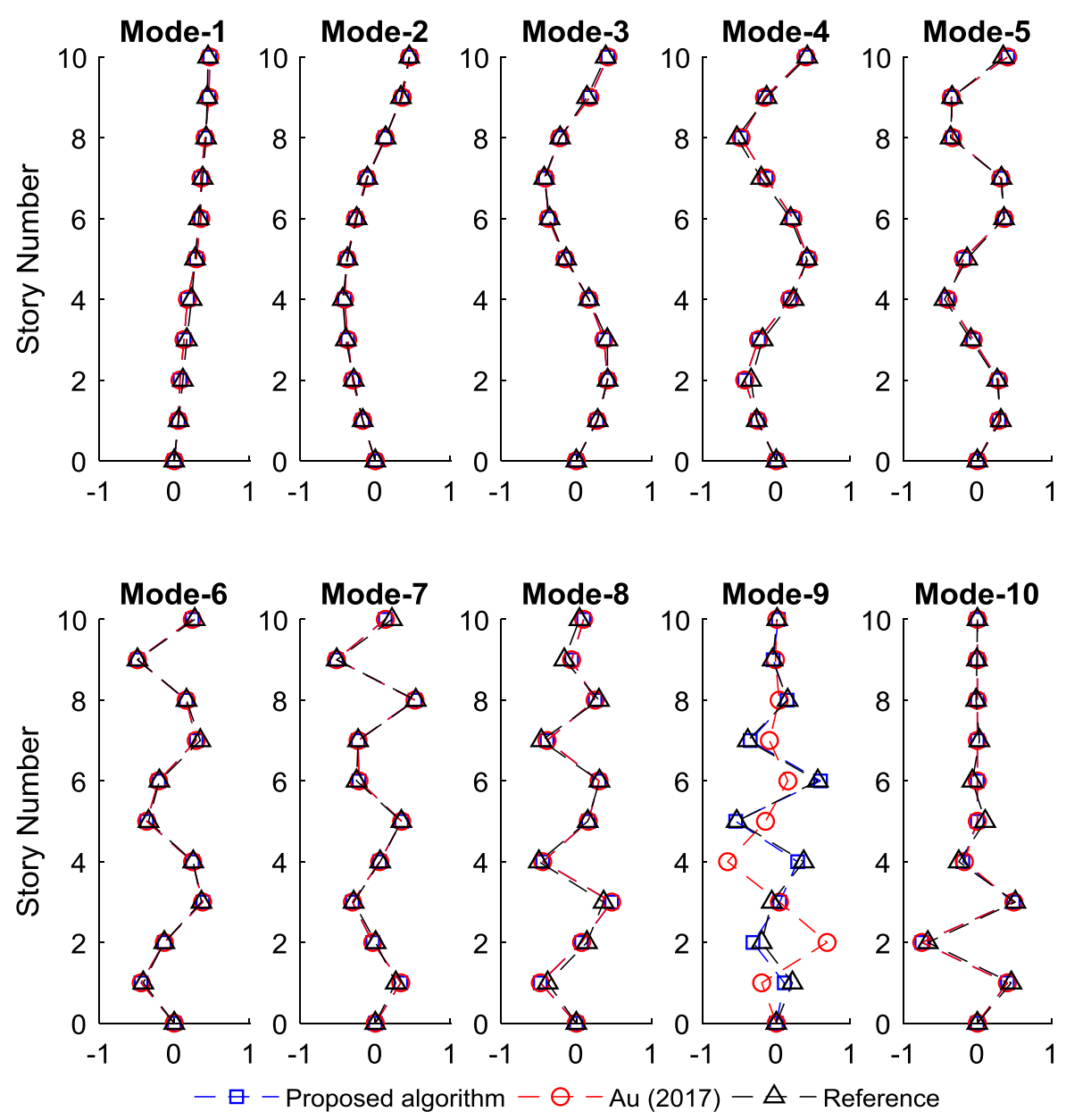

Fig. 9. Assembled mode shapes for Case-II.

Table 5

Calculated MAC values for Case I and II.

\begin{tabular}{|c|c|c|c|c|c|c|}
\hline \multirow[t]{2}{*}{ Mode number } & \multicolumn{3}{|l|}{ Case-I } & \multicolumn{3}{|l|}{ Case-II } \\
\hline & MAC1 & MAC2 & EMAC & MAC1 & MAC2 & EMAC \\
\hline 1 & 0.9963 & 0.9962 & 0.9998 & 0.9980 & 0.9978 & 0.9991 \\
\hline 2 & 0.9932 & 0.9934 & 0.9993 & 0.9960 & 0.9954 & 0.9990 \\
\hline 3 & 0.9915 & 0.9927 & 0.9996 & 0.9986 & 0.9970 & 0.9994 \\
\hline 4 & 0.9972 & 0.9912 & 0.9992 & 0.9945 & 0.9929 & 0.9993 \\
\hline 5 & 0.9950 & 0.9933 & 0.9983 & 0.9972 & 0.9967 & 0.9988 \\
\hline 6 & 0.9801 & 0.9752 & 0.9882 & 0.9852 & 0.9827 & 0.9857 \\
\hline 7 & 0.9856 & 0.9822 & 0.9906 & 0.9825 & 0.9809 & 0.9860 \\
\hline 8 & 0.9741 & 0.9695 & 0.9873 & 0.9748 & 0.9737 & 0.9843 \\
\hline 9 & 0.9665 & 0.3847 & 0.9783 & 0.9763 & 0.1861 & 0.9805 \\
\hline 10 & 0.9693 & 0.9659 & 0.9935 & 0.9848 & 0.9658 & 0.9835 \\
\hline
\end{tabular}


sented in Fig. 3. According to the results, the identified signal-to-noise levels show a good convergence with the analytical values especially for Setups 1, 2, and 3. Since the noise level is considerably large in setup 4, the variability of the identified signal-to-noise ratios is increased. However, the results are reasonable when compared to the analytical values for Setup 4.

Identified global mode shape vectors for the first five modes are presented in Fig. 4. The identified mode shapes well match with the analytical values, and the MAC values between the identified and analytical mode shapes are obtained as $0.9997,0.9998,1.000,0.9999,0.9998$, respectively. In addition, the EMAC values of the identified values are calculated as 0.9995, 0.9997, 1.000, 1.000 and 1.000, respectively.

Bayesian methods (BSDA and BFFTA) give reasonable results for the modal parameters under large noise effect since the modelling error and environmental noise effects are considered as prediction error in the methodology. In the case of multiple measurement setups, the main problem resides in the assembly of local mode shapes that have different identification qualities (or uncertainties). In this study, the mode shapes are assembled by using the uncertainty information of local spectrum variables without regarding the local mode shape information. According to the results of this numerical example, the quality of the identified mode shapes is observed to be considerably large in spite of the significant variability in the noise levels among different setups.

\subsection{Experimental study: laboratory shear frame}

A ten-story shear frame shown in Fig. 5 is measured with two different multiple setup configurations (see Table 3). These cases are considered in order to see the effect of sensor configuration on the assembly procedure. Here, Case-I represents a fixed reference sensor placement, while Case-II represents a roving reference sensor placement configuration. Small amplitude acceleration responses are acquired under ambient excitation for both cases. For comparison purposes, the given shear frame was measured under adequately large amplitude ambient excitation and the obtained frequencies, damping ratios and modal shape results are used as reference values.

In the laboratory experiments, piezo-electric accelerometers are used which are defined with $1000 \mathrm{mV} / \mathrm{g}$ sensitivity and $11.4 \mu \mathrm{g} /(\mathrm{Hz})^{0.5}$ spectral noise density. The measurement system consists of a laptop computer with a $1.5 \mathrm{GHz}$ single CPU and Linux operating system, a 16 channel USBDUX-Sigma data acquisition box with 24 bit analog to digital conversion, a first order analog lowpass filter with a cut-off frequency at $120 \mathrm{~Hz}$ for each channel, and a constant current supply for the
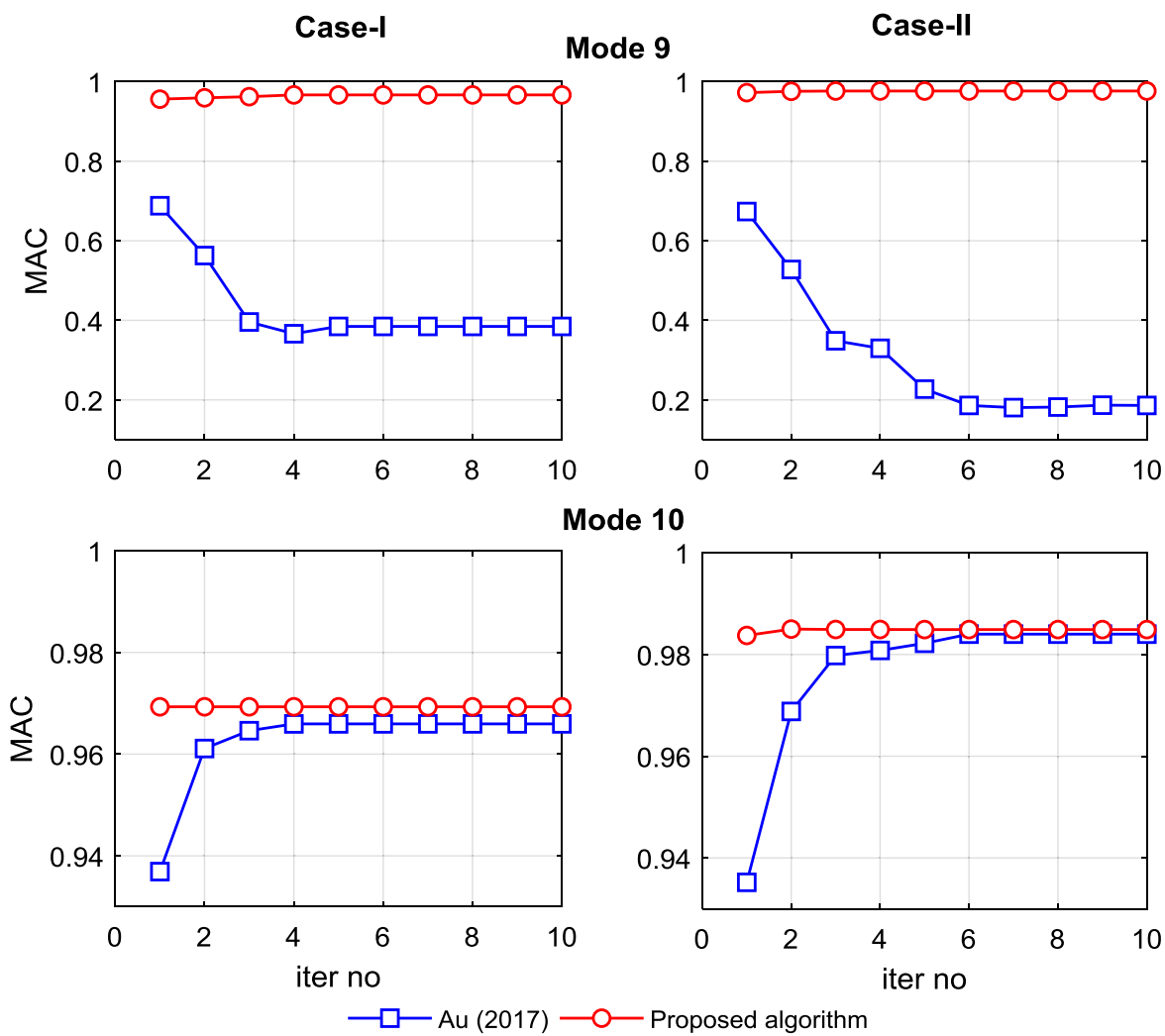

Fig. 10. Variations in MAC values with respect to reference mode shapes versus the number of iterations. 
accelerometers. The acceleration responses are recorded in the weak direction of the buildings and all measurements of groups are acquired in different times with $1000 \mathrm{~Hz}$. sampling frequency and 5 min duration.

A manual selection of the bandwidth for possible modes requires a visual inspection of the frequency response data. For each measurement setup, the maximum root maximum singular value spectrums for Case-I and II are obtained by windowing (with 600 segments), and they are presented in Fig. 6, respectively. The selected bandwidths of possible modes are marked in the figures by lateral error bars.

The average values of the MPV for natural frequencies and damping ratios obtained at each setup together with coefficients of variation are presented in Table 4 for Case-I and II. According to the results, the maximum c.o.v. remains less than $1 \%$ for each case. For damping ratios, standard deviations show relatively large variations among different setups. These variations are the result of the setup-to-setup variability instead of identification uncertainty. Natural frequencies and damping ratios are expected to be invariant for each setup. To the contrary, the spectral density of modal excitation and prediction error may show significant variations among different setups due to the possible variations in excitation levels. As a result, a decrease in signal strength is observed for larger modes. To illustrate this case, average modal signal-to-noise ratios and their setup-to-setup variations are presented in Fig. 7.

In Fig. 7, the variations in the estimated signal-to-noise ratios, $\gamma_{k i \text { max }}=\bar{S}_{i} /\left(4 \xi_{i}^{2} S_{e i}\right)$, are presented. For both cases, setup-tosetup variability of signal-to-noise ratio is remarkably large for all modes. This variability may not affect the identification

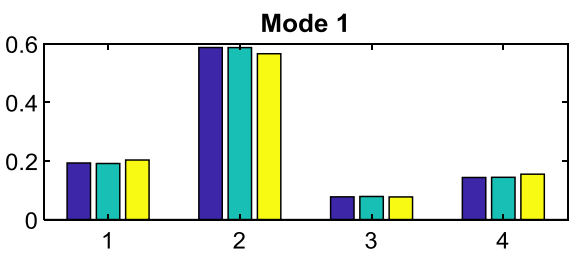

Mode 3



Mode 5


Mode 9

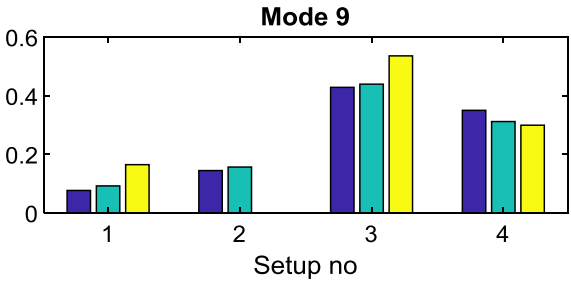

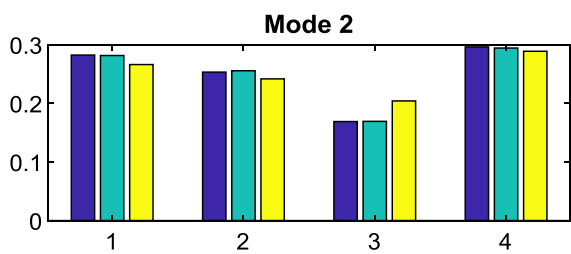

Mode 4

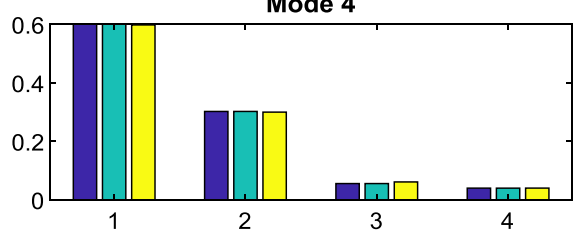

Mode 6

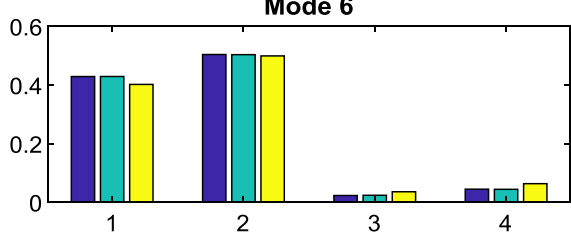

Mode 8
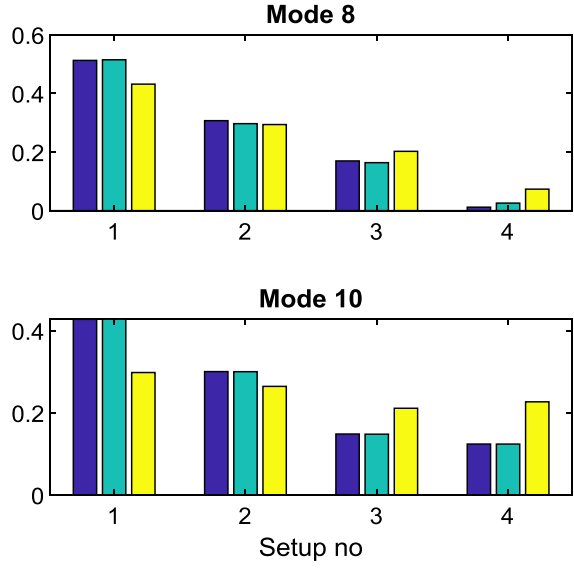

Initial

Final

Asymptotic

Fig. 11. Calculated setup weights for Case-I. 
quality in lower modes due to the relatively higher signal quality. For higher modes (Modes 6-10), however, this variability may cause significant errors in the identification process due to the smaller signal-to-noise effect.

Assembled mode shapes for Case-I and II are presented in Figs. 8 and 9, respectively. The iterations of algorithms are stopped as the MAC value between the current and previous steps is larger than 0.9999. According to the results, it is seen that the assembled mode shapes by using the presented methodology are similar for the proposed methodology and the algorithm by Au [19]. The ninth mode obtained by using the algorithm by Au [19], however, does not meet the reference mode shape. The reason of this fact resides on the initial guess proposed by $\mathrm{Au}$ [19] which is quite far away from the optimal value. The MAC values between the identified and reference mode shapes are presented in Table 5. Here, MAC1 and MAC2 indicate the MAC values for the mode shapes obtained by the proposed methodology and the algorithm by Au [19], respectively, with respect to reference mode shapes. Both procedures give similar results except for the ninth mode.

Fig. 10 presents the MAC values of the mode shapes that are obtained at each iteration step for Mode 9 and 10 with respect to reference mode shapes. The lower modes show no significant variance, and therefore they are not shown. It is seen that the quality of the initial guess of the proposed algorithm gives more reasonable results when compared to the results of the algorithm by Au [19]. The reason is considered to be caused by the large signal-to-noise asymptotic behavior assumption for the initial guess by Au [19]. Since the higher modes are subjected to less level of excitation, the initial guess by using the
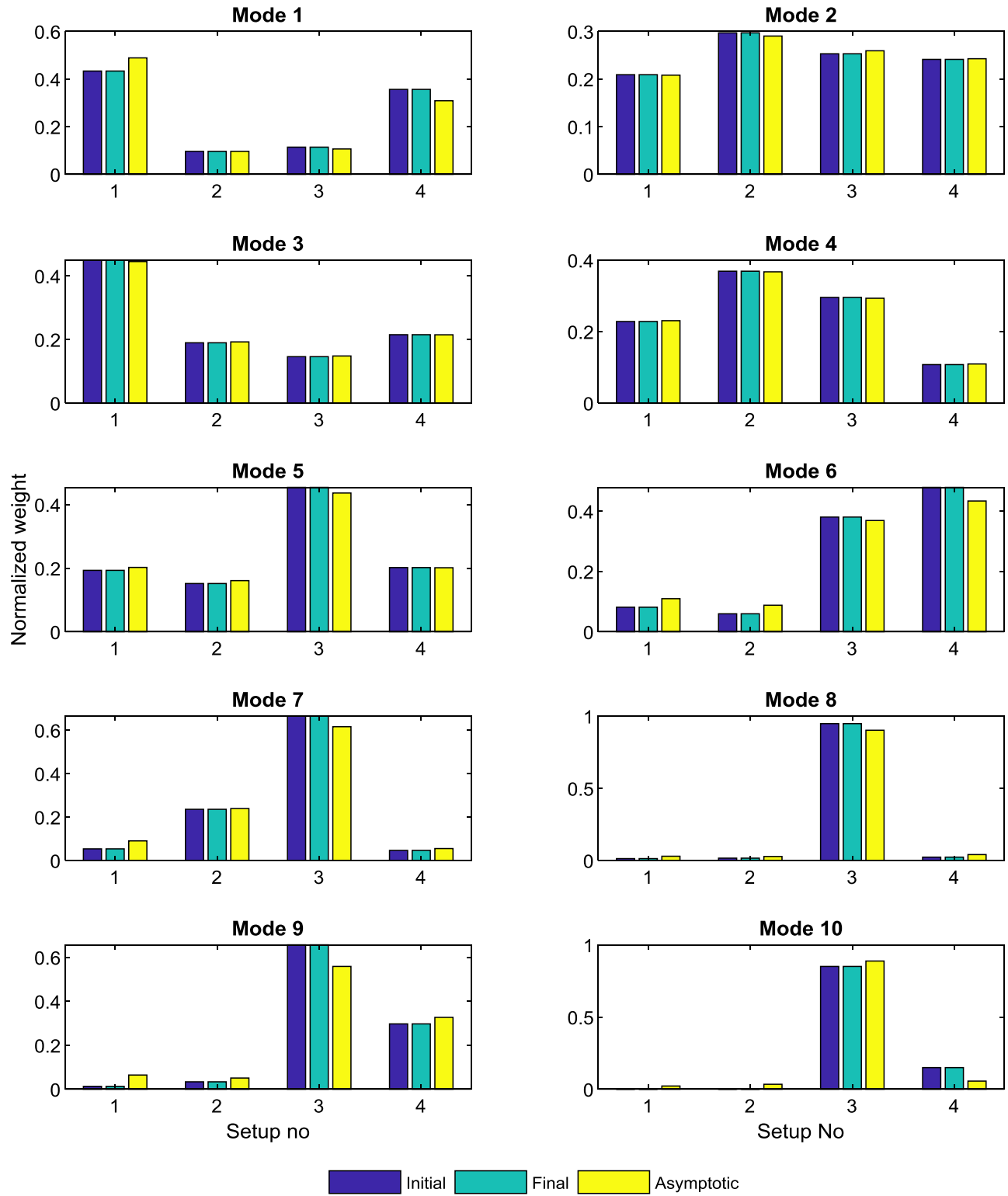

Fig. 12. Calculated setup weights for Case-II. 
large signal-to-noise asymptotic behavior may significantly diverge from the actual value. Figs. 11 and 12 present the calculated weights for the initial and final mode shapes by the proposed methodology and the asymptotic weight proposed by $\mathrm{Au}$ [19]. It can be seen that the weights for modes 1-8 are in the same range. The asymptotic weights for modes 9

\section{Sensor locations for deck}



Fig. 13. Schematic representation of Z24 Bridge and sensor layouts $[25,26]$. 
and 10, however, are quite different from the proposed algorithm, which is considered to be the cause of the difference in the ninth mode shape.

\subsection{Benchmark study: Z24 bridge}

The former Z24 Bridge in Switzerland is investigated in this section. The bridge is used in a benchmark study under the COST-F European Network project [26]. The bridge passes over the Bern-Zürich highway, and connects the two towns of Utzenstorf and Koppigen. It is composed of three spans with lengths of 14, 30, and $14 \mathrm{~m}$ as it is illustrated in Fig. 13.

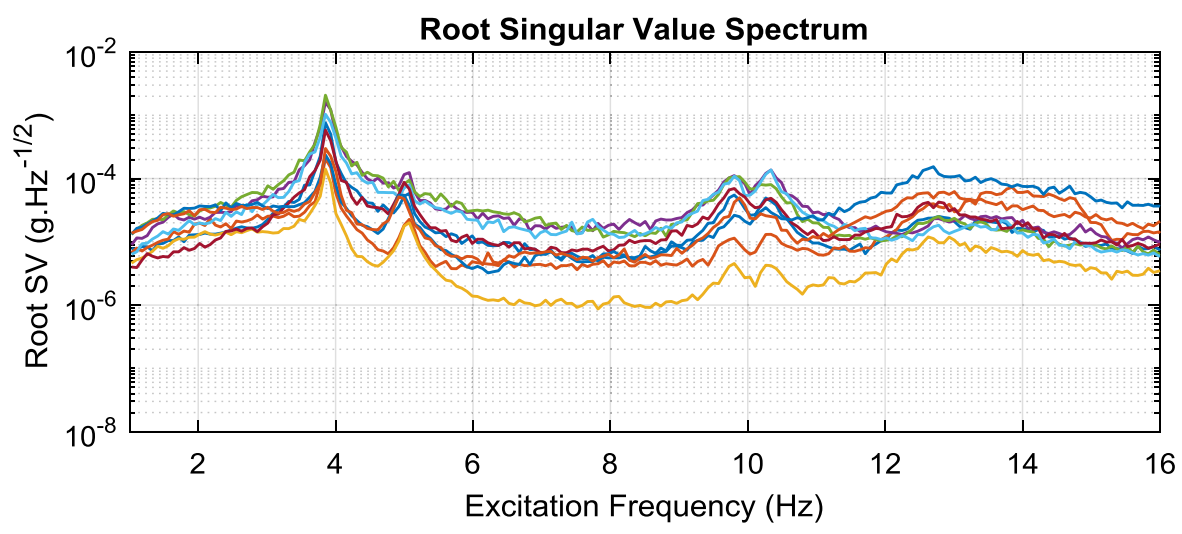

Fig. 14. Maximum root-singular value spectra for all setups.

Table 6

Estimated frequencies and damping ratios.

\begin{tabular}{|c|c|c|c|c|c|c|c|c|}
\hline \multirow{2}{*}{$\begin{array}{l}\text { Mode } \\
\text { Number }\end{array}$} & \multicolumn{4}{|l|}{$f(\mathrm{~Hz})}$. & \multicolumn{4}{|l|}{$\xi(\%)$} \\
\hline & $\begin{array}{l}\text { Peeters and Ventura } \\
{[24]}\end{array}$ & $\begin{array}{l}\text { Reynders et al. } \\
{[25]}\end{array}$ & $\begin{array}{l}\text { Present } \\
\text { Study }\end{array}$ & $\begin{array}{l}\text { c.o.v. } \\
(\%)\end{array}$ & $\begin{array}{l}\text { Peeters and Ventura } \\
{[24]}\end{array}$ & $\begin{array}{l}\text { Reynders et al. } \\
{[25]}\end{array}$ & $\begin{array}{l}\text { Present } \\
\text { Study }\end{array}$ & $\begin{array}{l}\text { C.o.v. } \\
(\%)\end{array}$ \\
\hline 1 & 3.86 & 3.86 & 3.85 & 0.30 & 0.90 & 0.80 & 0.92 & 24.54 \\
\hline 2 & 4.90 & 4.90 & 4.89 & 0.41 & 1.40 & 1.40 & 1.36 & 19.34 \\
\hline 3 & 9.77 & 9.76 & 9.77 & 0.25 & 1.30 & 1.40 & 1.19 & 27.59 \\
\hline 4 & 10.30 & 10.30 & 10.32 & 0.87 & 1.40 & 1.30 & 1.94 & 54.40 \\
\hline 5 & 12.50 & 12.42 & 12.53 & 1.15 & 2.50 & 2.80 & 3.18 & 36.84 \\
\hline 6 & 13.20 & 13.22 & 13.22 & 0.86 & 3.00 & 3.40 & 3.05 & 37.45 \\
\hline
\end{tabular}
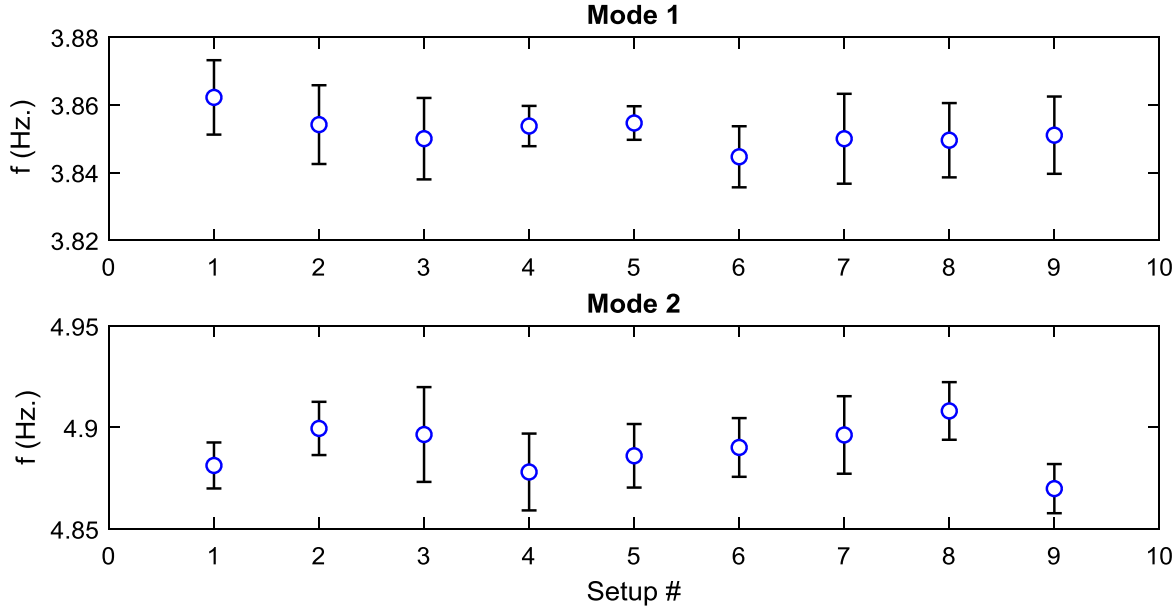

Fig. 15. Identified frequencies with representative statistics ( \pm standard deviations). 
In the benchmark study, the Z24 Bridge is measured at 152 points under ambient and forced vibrations by KU LEUVEN Structural Mechanics division between 1998 and 2000. Nine measurement setups with three reference points are conducted to obtain a complete measurement of the bridge. The schematic representation of the sensor layout for the deck and piers are shown in Fig. 13. The collected data for seventeen different cases is available on the website https://bwk.kuleuven.be/bwm/ z24. Among these, the third reference measurement is considered in this study.

The average maximum root singular value spectrum of reference measurement- 3 by using 13,000 windows is presented in Fig. 14. Here, the first five modes are easily perceptible. The sixth mode is not excited in all setups. It is visible in the second, third and seventh setups, only. In addition, it is seen that the visible modes are well separated. A similar trend is observed in previous studies by Peeters and Ventura [24], and Reynders et al. [25].

The calculated natural frequencies and damping ratios are presented in Table 6 and compared with the results by Peeters and Ventura [24], and Reynders et al. [25]. The identified results are the mean values obtained as the average of the MPVs for all setups. In addition, the posterior coefficient of variations including the setup-to-setup variability and identification are presented in Table 6 as well. The calculated MPVs of frequency and damping ratio are compatible with results by the previous studies [24,25]. The largest relative difference is less than $2 \%$. Variations in the most probable frequency and damping ratios are presented in Figs. 15 and 16, respectively. Here, error bars show the posterior standard deviations. In addition, due

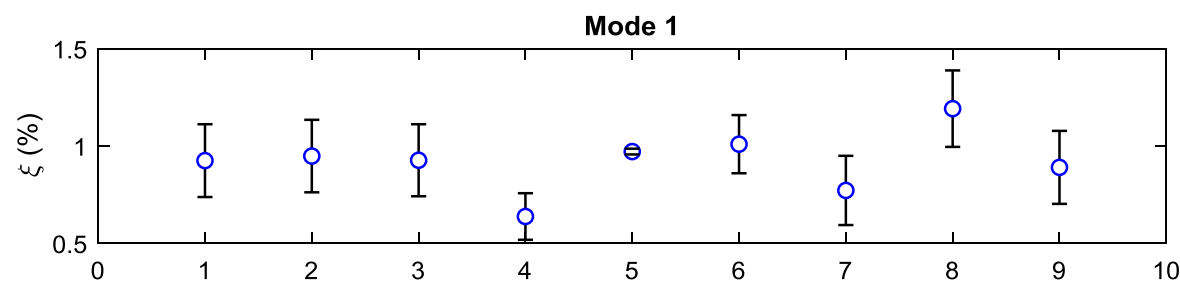

Mode 2

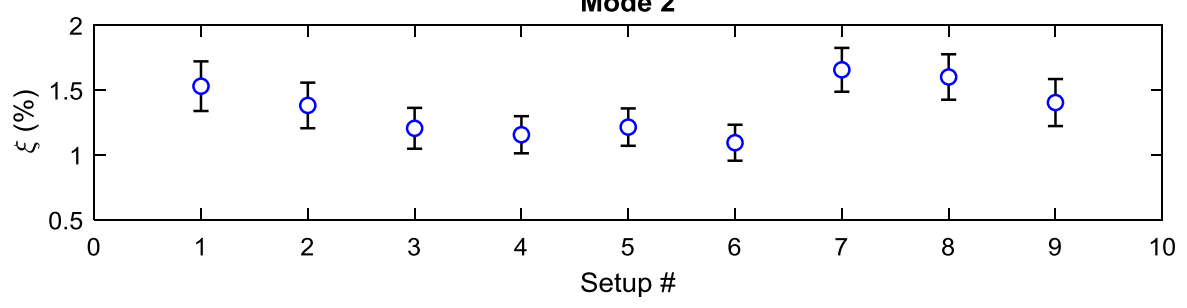

Fig. 16. Identified damping ratios with representative statistics ( \pm standard deviations).

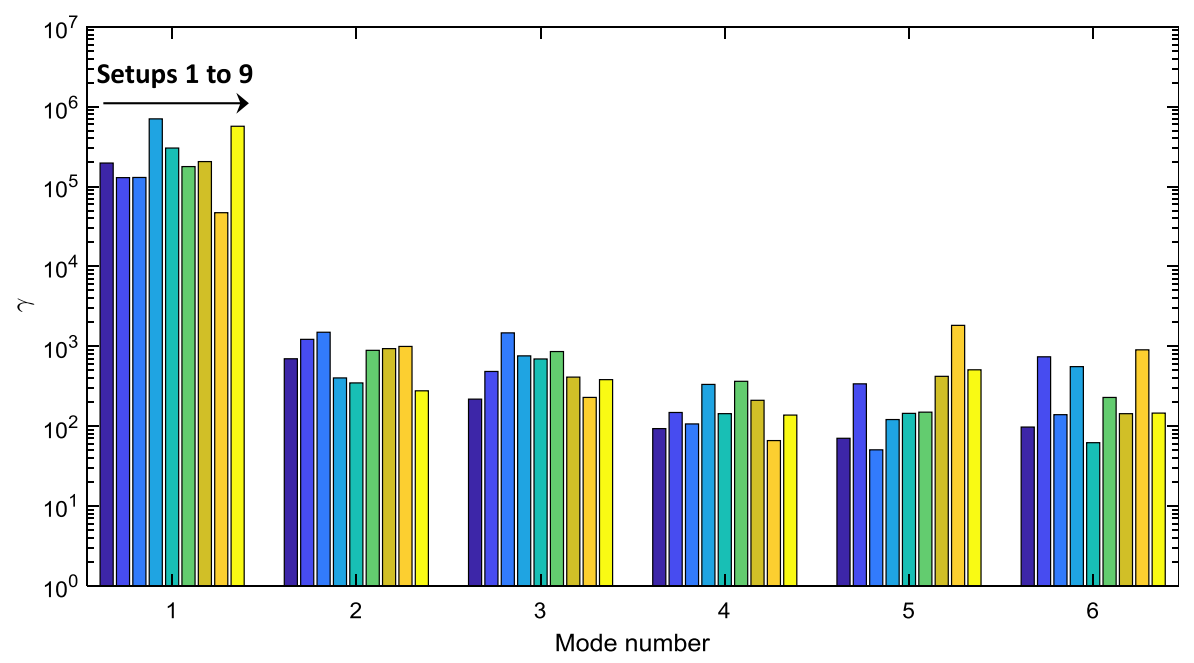

Fig. 17. Variations in the identified signal-to-noise ratios among different setups. 




$\underline{\text { Mode-1 }}$


Mode-2

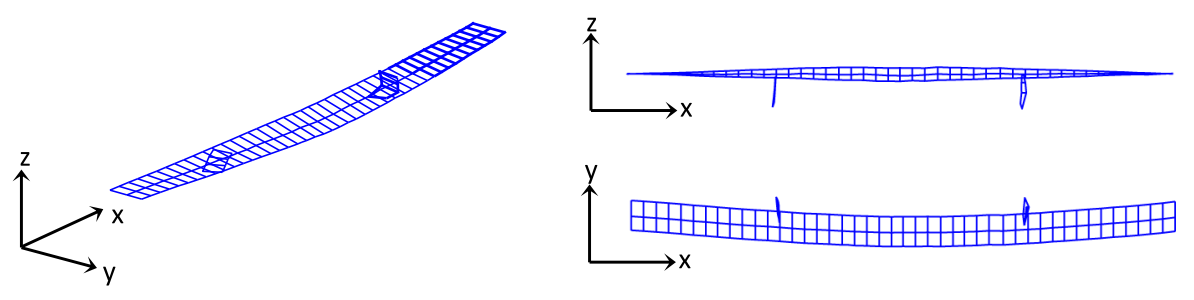

$\underline{\text { Mode-3 }}$

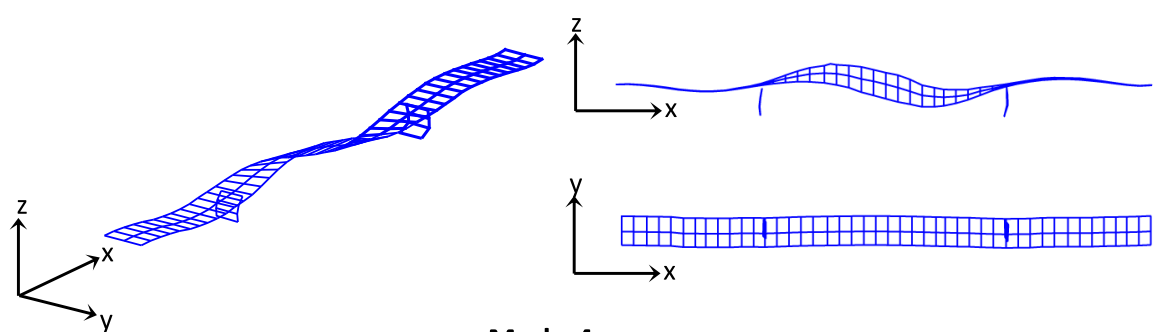

Mode-4

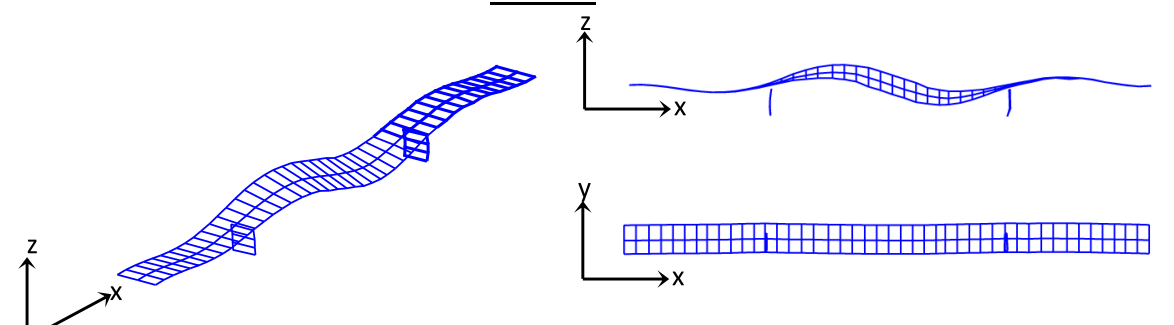

Mode-5



$\underline{\text { Mode- } 6}$

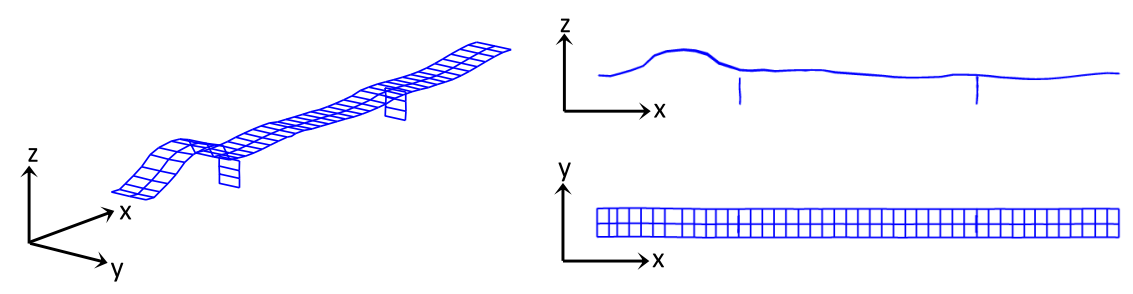

Fig. 18. Assembled mode shapes by the proposed algorithm. 
Table 7

MAC values for the estimated mode shapes.

\begin{tabular}{llll}
\hline Mode number & EMAC & MAC $_{\mathrm{as}}$ & $\mathrm{MAC}_{\mathrm{ref}}$ \\
\hline 1 & 1.0000 & 1.0000 & 1.0000 \\
2 & 1.0000 & 0.9999 & 1.0000 \\
3 & 1.0000 & 1.0000 & 1.0000 \\
4 & 1.0000 & 0.8826 & 1.0000 \\
5 & 1.0000 & 0.9992 & 0.9999 \\
6 & 1.0000 & 0.9991 & 1.0000 \\
\hline
\end{tabular}
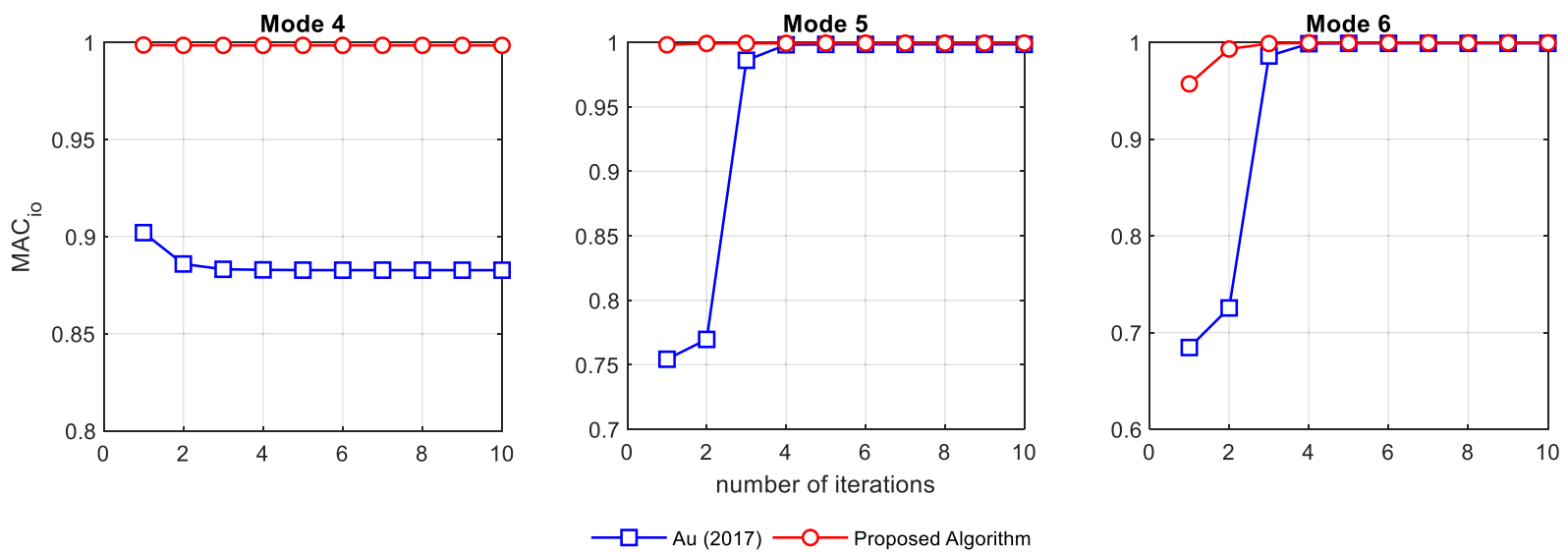

Fig. 19. Variations in $M A C_{i, 0}$ values with respect to number of iterations.

to the large signal-to-noise ratio, the signal quality is observed to be good for all setups according to the results presented in Fig. 17.

Fig. 18 presents the assembled global mode shapes by the proposed algorithm. The mode shapes for the first six modes are well identified and seem compatible with previous studies [24,25]. The algorithm by Au [19] gives similar results, and the first three modes are directly identified without iteration. Table 7 presents the calculated MAC values between the proposed methodology and the algorithm by $\mathrm{Au}$ [19]. Here, $M A C_{\text {as }}$ and $M A C_{\text {ref }}$ represent the MAC values between the final mode shapes by the proposed method, and the initial guess and final mode shapes obtained from the algorithm by Au [19], respectively. It is seen that the results are similar for both methodologies. The fourth mode, however, shows a difference about $12 \%$ between the initial guess by $\mathrm{Au}[19]$ and the final mode shapes.

Convergence of MAC values with respect to the iteration number is presented in Fig. 19. Here, MAC $\mathrm{C}_{\mathrm{i}, 0}=\mathrm{MAC}_{\mathrm{value}}$ between the mode shapes at the $i^{\text {th }}$ iteration and the initial guess of each algorithms. According to Fig. 19 the estimation of the 4th mode shape diverges down to a MAC value of 0.88 from the initial guess with the algorithm by Au [19]. Further, estimations of mode five and six, initially show a significant divergence from the initial guess which is cancelled out in a few numbers of iteration. The MAC values for the proposed algorithm, however, indicates that nearly no change takes place during iteration. The proposed algorithm reaches to this optimal global mode shape without iteration, and therefore it can be concluded that the initial guess of the proposed algorithm is more feasible. The difference between the initial guess by Au [19] and the proposed algorithm lies in the involvement of spectrum parameters in the calculation of initial guess by the proposed algorithm.

In order to figure out the reason of the divergence in the MAC values, especially for mode 4 , the calculated normalized weights of the setups in the global mode shapes are presented in Fig. 20. For mode 4, it can be seen that the asymptotic weight is much lower than the weights calculated by the proposed methodology at setup 3 . This case is not the reason, but it may be considered to be in parallel with the divergence in the MAC value as shown in Fig. 19. 

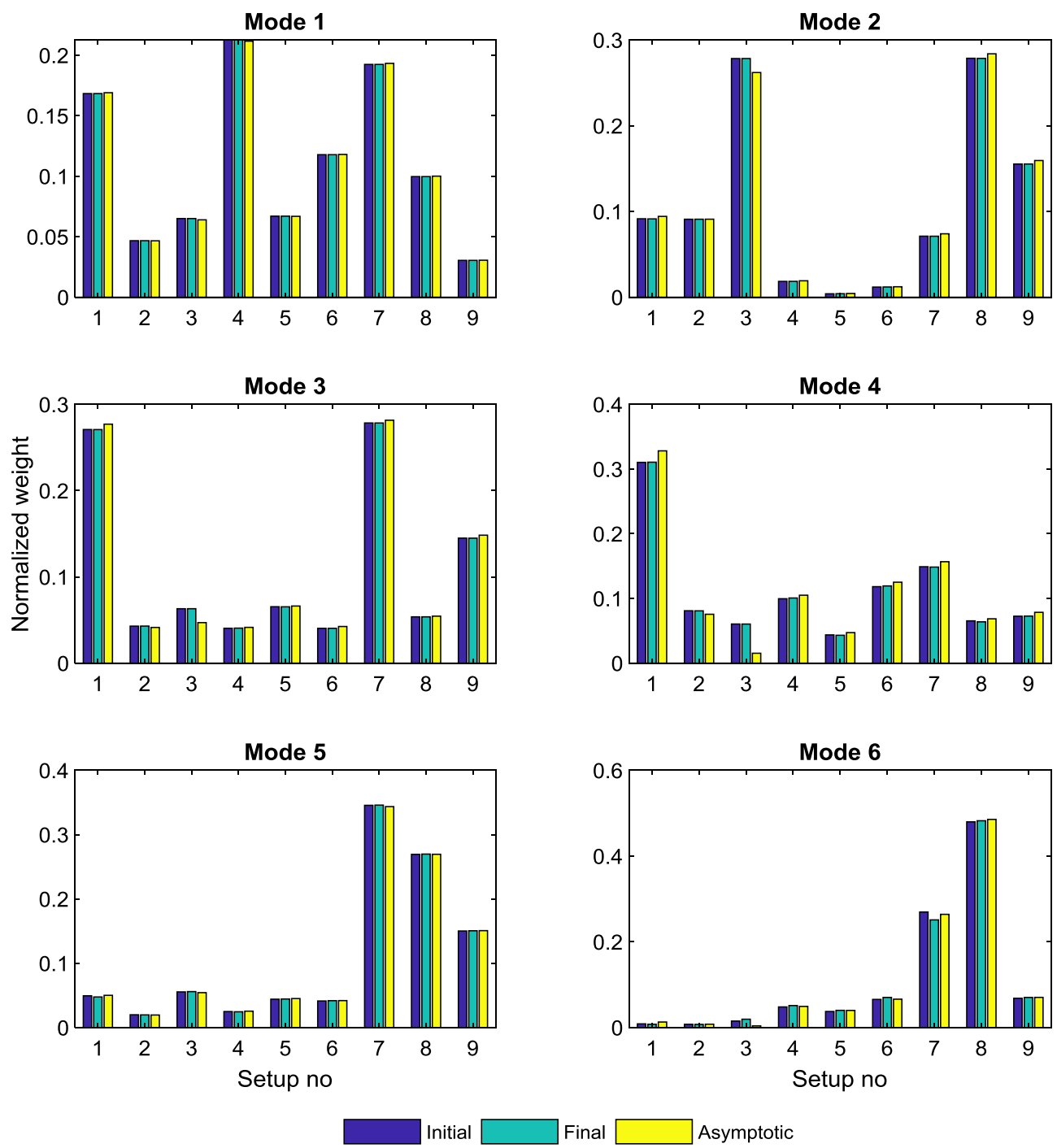

Fig. 20. Variations in calculated setup weights.

\section{Conclusions}

In this study, an alternative mode shape assembly algorithm based on the two-stage BFFTA is presented, and the uniqueness of the solution is verified analytically by using two main methodologies, available in the literature. These are based on the direct estimation of the global mode shape vector incorporating the FFT data or assembling the identified local mode shapes by using the Gaussian approximation, respectively. The proposed methodology is implemented to a ten-story laboratory shear frame and a benchmark study, and the obtained results are compared to the algorithm by Au [19]. The general conclusions are summarized below.

- The proposed procedure considers that each setup is weighted by its Hessian matrix (for local mode shape) and the local mode shape information conducted with spectrum parameters is embedded in this matrix. Thus, the global mode shape vector is obtained by assembling the local Hessian matrices and it is not necessary to obtain the optimal local mode shape vectors for each setup.

- In the literature, the initial guess for global mode shape vector assumes large signal-to-noise ratio and neglects the effect of data quality. If the signal-to-noise ratio is not adequately large for all or a few setups, however, the initial guess may significantly diverge from the optimal mode shape. The proposed application, on the other hand, uses the two-stage approach leading to a more reasonable initial guess for global mode shapes which increases the convergence speed during iteration. 
- When the data quality is well in each setup, the identified mode shapes shows no significant variance among any method that is being used. According to the results by the ten- story shear frame study, however, it is seen that the quality of identified mode shapes by the proposed algorithm is improved when the signal-to-noise ratio is low.

\section{Acknowledgments}

The authors would like to thank to KU LEUVEN Structural mechanics division for sharing the measurement data of the Z24 Bridge.

\section{References}

[1] L.S. Katafygiotis, K.V. Yuen, Bayesian spectral density approach for modal updating using ambient data, Earthquake Eng. Struct. Dyn. 30 (2001) $1103-$ 1123.

[2] K.V. Yuen, L.S. Katafygiotis, Bayesian Fast Fourier Transform Approach for modal updating using ambient data, Adv. Struct. Eng. 6 (2) (2003) 81-95.

[3] S.K. Au, Fast Bayesian FFT method for ambient modal identification with separated modes, J. Eng. Mech. 137 (3) (2011) $214-226$.

[4] S.K. Au, Fast Bayesian ambient modal identification in the frequency domain, Part I: Posterior most probable value, Mech. Syst. Signal Process. 26 (2012) 60-75.

[5] S.K. Au, F.L. Zhang, Ambient modal identification of a primary-secondary structure by Fast Bayesian FFT method, Mech. Syst. Signal Process. 28 (2012) 280-286.

[6] S.K. Au, F.L. Zhang, Y.C. Ni, Bayesian operational modal analysis: theory, computation, practice, Comput. Struct. 126 (2013) 3-14.

[7] S.K. Au, Insights on the Bayesian spectral density method for operational modal analysis, Mech. Syst. Signal Process. 66-67 (2016) 1-12.

[8] F.L. Zhang, Y.C. Ni, S.K. Au, H.F. Lam, Fast Bayesian approach for modal identification using free vibration data, Part-I: most probable value, Mech. Syst. Signal Process. 70-71 (2016) 209-220.

[9] Y.C. Ni, F.L. Zhang, H.F. Lam, S.K. Au, Fast Bayesian approach for modal identification using free vibration data, Part II: Posterior uncertainty and application, Mech. Syst. Signal Process. 70-71 (2016) 221-244.

[10] W.J. Yan, L.S. Katafygiotis, A two-stage fast Bayesian spectral density approach for ambient modal analysis. Part I: Posterior most probable value and uncertainty, Mech. Syst. Signal Process. 54-55 (2015) 139-155.

[11] Z. Li, M.Q. Feng, L. Luo, D. Feng, X. Xu, Statistical analysis of modal parameters of a suspension bridge based on Bayesian spectral density approach and SHM data, Mech. Syst. Signal Process. 98 (2018) 352-367.

[12] Y. Ni, X. Lu, W. Lu, Operational modal analysis of a high-rise multi-function building with dampers by a Bayesian approach, Mech. Syst. Signal Process. 86 (2017) 286-307.

[13] H.-F. Lam, F.-L. Zhang, Y.-C. Ni, J. Hu, Operational modal identification of a boat-shaped building by a Bayesian approach, Eng. Struct. 138 (2017) 381393.

[14] P. Liu, F.L. Zhang, P.Y. Lian, Dynamic characteristic analysis of two Adjacent multi-grid composite wall structures with a seismic joint by a Bayesian approach, J. Earthquake Eng. 20 (2016) 1295-1321.

[15] M. Döhler, P. Andersen, L. Mevel, Data merging for multi-setup operational modal analysis with data-driven SSI, Structural Dynamics, Volume 3. Conference Proceedings of the Society for Experimental Mechanics Series, Springer, New York, NY, 2011.

[16] M. Döhler, E. Reynders, F. Magalhães, L. Mevel, G.D. Roeck, Á. Cunha, Pre- and post-identification merging for multi-setup OMA with covariance-driven SSI, Dynamics of Bridges, Volume 5. Conference Proceedings of the Society for Experimental Mechanics Series, Springer, New York, NY, 2011.

[17] S.K. Au, Assembling mode shapes by least squares, Mech. Syst. Signal Process. 25 (2011) 163-179.

[18] S.K. Au, F.L. Zhang, Fast Bayesian ambient modal identification incorporating multiple setups, J. Eng. Mech. 138 (7) (2012) 800-815.

[19] S.K. Au, Operational Modal Analysis: Modeling, Bayesian Inference, Uncertainty Laws, E-Book, Springer, Singapore, 2017.

[20] W.J. Yan, L.S. Katafygiotis, A two-stage fast Bayesian spectral density approach for ambient modal analysis. Part II: Mode shape assembly and case studies, Mech. Syst. Signal Process. 54-55 (2015) 156-171.

[21] S.K. Au, Y.L. Xie, Calculation of Hessian under constraints with applications to Bayesian system identification, Comput. Methods Appl. Mech. Eng. 323 (2017) 373-388.

[22] F.L. Zhang, S.K. Au, On assessing the posterior mode shape uncertainty in ambient modal identification, Probab. Eng. Mech. 26 (2011) 427-434.

[23] F.L. Zhang, S.K. Au, Probabilistic model for modal properties based on operational modal analysis, ASCE-ASME J. Risk Uncertainty Eng. Syst., Part A: Civ. Eng. 2 (2016) B4015005.

[24] B. Peeters, C.E. Ventura, Comparative study of modal analysis techniques for bridge dynamic characteristics, Mech. Syst. Signal Process. 17 (2003) $965-$ 988.

[25] E. Reynders, J. Houbrechts, G. De Roeck, Fully automated modal analysis, Mech. Syst. Signal Process. 29 (2012) $228-250$.

[26] E. Reynders, G. De Roeck, Reference-based combined deterministic-stochastic subspace identification for experimental and operational modal analysis, Mech. Syst. Signal Process. 22 (2008) 617-638. 